\title{
Noise-assisted estimation of attractor invariants
}

\author{
Juan F. Restrepo ${ }^{1,2, *}$ and Gastón Schlotthauer ${ }^{1,2,3}$ \\ ${ }^{1}$ Laboratorio de Señales y Dinámicas no Lineales, Facultad de Ingeniería, Universidad Nacional de Entre Ríos, Argentina \\ ${ }^{2}$ National Scientific and Technical Research Council (CONICET), Argentina \\ ${ }^{3}$ Centro de Investigaciones y Transferencia de Entre Ríos (CITER), Argentina
}

(Received 19 March 2016; published 12 July 2016)

\begin{abstract}
In this article, the noise-assisted correlation integral (NCI) is proposed. The purpose of the NCI is to estimate the invariants of a dynamical system, namely the correlation dimension $(D)$, the correlation entropy $\left(K_{2}\right)$, and the noise level $(\sigma)$. This correlation integral is induced by using random noise in a modified version of the correlation algorithm, i.e., the noise-assisted correlation algorithm. We demonstrate how the correlation integral by Grassberger et al. and the Gaussian kernel correlation integral (GCI) by Diks can be thought of as special cases of the NCI. A third particular case is the $U$-correlation integral proposed herein, from which we derived coarse-grained estimators of the correlation dimension $\left(D_{m}^{U}\right)$, the correlation entropy $\left(K_{m}^{U}\right)$, and the noise level $\left(\sigma_{m}^{U}\right)$. Using time series from the Henon map and the Mackey-Glass system, we analyze the behavior of these estimators under different noise conditions and data lengths. The results show that the estimators $D_{m}^{U}$ and $\sigma_{m}^{U}$ behave in a similar manner to those based on the GCI. However, for the calculation of $K_{2}$, the estimator $K_{m}^{U}$ outperforms its GCI-based counterpart. On the basis of the behavior of these estimators, we have proposed an automatic algorithm to find $D, K_{2}$, and $\sigma$ from a given time series. The results show that by using this approach, we are able to achieve statistically reliable estimations of those invariants.
\end{abstract}

DOI: 10.1103/PhysRevE.94.012212

\section{INTRODUCTION}

Chaotic phenomena arise naturally in many fields of science. They can be observed in the nonlinear dynamics of biological systems [1] as well as in the evolution of economic data [2]. In the past few decades, scientists have focused their efforts on developing mathematical concepts that allow us to understand and characterize the complex and unpredictable behavior of these deterministic phenomena. Two of those concepts are the correlation dimension $(D)$ and the correlation entropy $\left(K_{2}\right)$ proposed by Grassberger and Procaccia $[3,4]$. These invariants have been used in many applications, including ground motion analysis of earthquakes [5], characterization of high-layer coronal activity on the sun [6], classification of patients suffering from depression [7], etc. These invariants are estimated through the correlation integral, which is calculated by means of the correlation algorithm [8].

Despite the fact that the method of Grassberger and Procaccia, i.e., the standard correlation integral, has been proven to work well in low-dimensional attractors, it is almost useless when the time series coming from these systems are contaminated with noise [9-13]. The presence of noise causes the Grassberger-Procaccia method to overestimate the correlation dimension and the correlation entropy. This is because the approach assumes that the observed data represent a finite-dimensional set, and that there is no noise at all [12].

In experimental situations, in which there is always some level of noise, one has two options. The first is to try to reduce the noise from the time series (not always possible). However, the effect of a very small level of noise (2\%) can dramatically affect the estimation of invariants with the standard correlation integral [14]. The second option is to model the influence of

\footnotetext{
*Author to whom all correspondence should be addressed: jrestrepo@bioingenieria.edu.ar; Ruta Prov. 11 Km. 10. Oro VerdeEntre Ríos, Argentina.
}

noise in the scaling law $[11,15]$. Following this path, Diks proposed the Gaussian kernel correlation integral (GCI) [11]. In comparison with the standard correlation integral, the GCI not only has the ability to better estimate $D$ and $K_{2}$, but also the noise level $(\sigma)$. Estimating $\sigma$ is crucial to interpreting the results of an experiment, since it affects the estimations of the other invariants. In this sense, the researcher must be aware that if too much noise is present in the temporal series, caution must be taken in the interpretation of the results.

Unlike the Grassberger-Procaccia algorithm, this technique is relatively robust and correctly accounts for noise if that noise is Gaussian and additive. However, although it is much better than the Grassberger-Procaccia algorithm for estimating the correlation dimension, the Diks method still cannot estimate the correlation entropy, even in low-dimensional chaotic systems [12].

In this article, we propose a correlation integral that is induced by the use of noise in the correlation algorithm. The noise-assisted correlation integral (NCI) arises as a generalization of the standard correlation integral and also of the GCI. With the NCI we can also compute correlation integrals with kernel functions that would require numerical integration for its evaluation, given that this kernel function can be found to be the complementary cumulative density function of a random variable. We will explore a third particular case of the NCI, which we call the $U$-correlation integral (UCI). The particularity of this type of correlation integral is that it allows us to introduce information of the embedding dimension in the kernel function. Its main advantage relies on the convergence of the correlation entropy estimation. Moreover, from the UCI we have derived coarse-grained estimators of $D, K_{2}$, and $\sigma$. The behaviors of these estimators are tested with time series coming from low-dimensional systems under different noise levels and data lengths.

Section II is devoted to theoretical aspects reported in the literature that will be useful for the development of the article. 
In Sec. III we present the foundations of the NCI, and we present a particular case, the UCI, in Sec. IV. Two special cases of the UCI are studied in Secs. IV A and IV B. In Sec. V we derive the coarse-grained estimators based on the UCI for the correlation dimension (Sec. V A), the correlation entropy (Sec. V B), and the noise level (Sec. V C). Furthermore, we analyze their behavior under different conditions. Using these coarse-grained estimators, in Sec. VI we present an algorithm to automatically estimate system invariants. General remarks are given in Sec. VII, and the conclusions are presented in Sec. VIII.

\section{THEORY}

The correlation integral is the most important quantity used to calculate the correlation dimension and the correlation entropy of a dynamical system. The definition of the correlation integral involves phase-space vectors that are reconstructed from a scalar time series through an embedding method [15]. These phase-space vectors can be interpreted as random samples drawn from an underlying probability distribution (the natural measure on a system's attractor) [15]. Given two random vectors $\mathbf{x}$ and $\mathbf{y} \in \mathbb{R}^{m}$ chosen according to the natural measure $f_{X}(\mathbf{x})$ and a parameter $h>0$, a generalized definition of the correlation integral $G_{m}(h)$ is [11]

$$
\begin{aligned}
G_{m}(h) & =\iint g(\|\mathbf{x}-\mathbf{y}\| / h) f_{\mathbf{X}}(\mathbf{x}) f_{\mathbf{X}}(\mathbf{y}) d \mathbf{x} d \mathbf{y} \\
& =\int g(\tilde{z} / h) f_{\tilde{z}}(\tilde{z}) d \tilde{z},
\end{aligned}
$$

where $\tilde{z}=\|\mathbf{x}-\mathbf{y}\|$ is a measure of distance between the random vectors $\mathbf{x}$ and $\mathbf{y}$ (which will be assumed Euclidean), $f_{\tilde{z}}(\tilde{z})$ is its probability density function (PDF), and $m$ is the embedding dimension. From Eq. (1) it can be seen that the correlation integral is the expected value of a kernel function $g(\tilde{z} / h)$ that depends on a parameter $h$ (length scale). The standard correlation integral $C_{m}(h)$ is obtained by using the Heaviside step function $H(1-\tilde{z} / h)$ as a kernel [8],

$$
C_{m}(h)=\int H(1-\tilde{z} / h) f_{\tilde{z}}(\tilde{z}) d \tilde{z} .
$$

It is well known that the noise level of the time series affects the estimation of the correlation dimension [9-13]. In this sense, it is very important to model the influence of noise over this quantity, since if too much noise is present, then care must be taken in the interpretation of the results obtained by means of the correlation integral. The problem with the classical correlation integral is that its kernel function, the Heaviside step function [see Eq. (2)], makes it difficult to derive analytic functions to model the influence of noise in the estimation of the invariants [11]. To overcome this issue, Diks proposed the Gaussian kernel correlation integral $T_{m}(h)$, which in the absence of noise can be expressed as $[11,16]$

$$
T_{m}(h)=\int e^{-\tilde{z}^{2} / 4 h^{2}} f_{\tilde{z}}(\tilde{z}) d \tilde{z},
$$

where the kernel function is $g(\tilde{z} / h)=\exp \left(-\tilde{z}^{2} / 4 h^{2}\right)$. It has been shown that for fractal measures without noise, the standard correlation integral as well as the GCI scale as $[11,16,17]$

$C_{m}(h)=T_{m}(h)=\phi e^{-\tau m K_{2}} h^{D} \quad$ for $\quad m \rightarrow \infty, h \rightarrow 0$,

where $\phi \in \mathbb{R}$ is a normalization constant and $\tau$ is the embedding lag. This scaling behavior is observed if the kernel function $g(t)$ meets two conditions [11,18]: (i) it decreases monotonically for $t \geqslant 0$, and (ii) it decreases faster than a power function, i.e., $\lim _{h \rightarrow 0} h^{-p} g(t / h)=0$ for $t>0$ and any $p \geqslant 0$.

In real applications, the estimation of invariants is biased due to the finite length of the time series and/or due to the contamination with noise. The general idea to correct this bias [deviation from the scaling behavior of Eq. (4)] is to analytically model the noise influence over the scaling function. For example, when the time series is immersed in white Gaussian noise of variance $\sigma^{2}$, the GCI will scale as $[11,16,19]$

$$
\begin{aligned}
T_{m}(h)= & \phi h^{m}\left(h^{2}+\sigma^{2}\right)^{\frac{D-m}{2}} e^{-\tau m K_{2}} \\
& \text { for } m \rightarrow \infty, \sqrt{h^{2}+\sigma^{2}} \rightarrow 0 .
\end{aligned}
$$

Equation (4) is recovered when $\sigma \rightarrow 0$. In practice, $\sigma$ is fixed at a nonzero value, so the factor $\sqrt{h^{2}+\sigma^{2}}$ cannot tend to zero. However, it is expected that Eq. (5) holds in a suitable range of small $h$ values, provided that $\sigma$ is not too large. Corrected versions of the scaling law for the standard correlation integral [Eq. (2)] can be found in [20,21]. Moreover, there exist other approaches that are also useful [11,22,23].

Once the scaling model is chosen and the correlation integral is calculated, there are two main options to estimate $D$, $K_{2}$, and $\sigma$. The first one is to estimate these invariants through a nonlinear fitting of the scaling model $[11,16]$. This method has the drawback that it is highly dependent on the range of scale values selected to fit the model, and there is not a general consensus about the correct way to choose it. Additionally, to conclude that the dynamic arises from a deterministic system, it must be verified that the invariants do not depend on the scaling range. This is usually done by visual inspection of the "scaling regimes" [15].

The second option is to use coarse-grained estimators. These are explicit expressions for $D, K_{2}$, and $\sigma$ as functions of $m$ and $h$ [13]. Some examples of coarse-grained estimators are those derived by Nolte et al. [19],

$$
\begin{aligned}
& D_{m}^{T}(h)=\frac{d \ln T_{m}(h)}{d \ln h}-\frac{\left[m-\frac{d \ln T_{m}(h)}{d \ln h}\right] \Delta_{m}(h)}{1-\Delta_{m}(h)}, \\
& K_{m}^{T}(h)=\frac{1}{\tau}\left(\ln \frac{T_{m}(h)}{T_{m+1}(h)}+\frac{1}{2} \ln \left[1-\Delta_{m}(h)\right]\right),
\end{aligned}
$$

and

$$
\sigma_{m}^{T}(h)=\sqrt{\frac{h^{2} \Delta_{m}(h)}{1-\Delta_{m}(h)}},
$$

where $D_{m}^{T}, K_{m}^{T}$, and $\sigma_{m}^{T}$ are the coarse-grained estimators for $D, K_{2}$, and $\sigma$, respectively, and

$$
\Delta_{m}=\frac{d \ln T_{m+1}(h)}{d \ln h}-\frac{d \ln T_{m}(h)}{d \ln h} .
$$




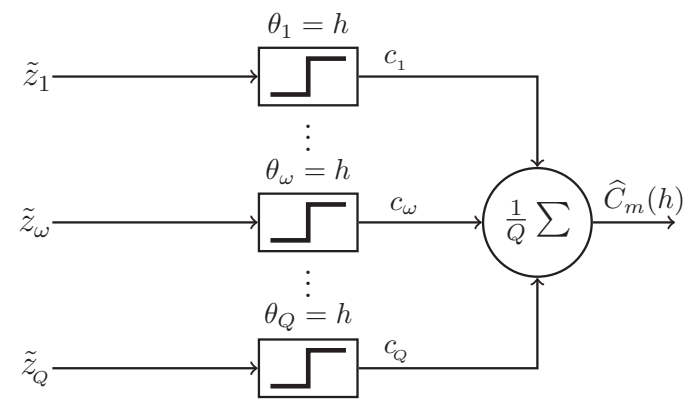

FIG. 1. Correlation algorithm: The distances $\tilde{z}_{\omega}$ between all pair of phase-space vectors are compared with a threshold $\theta_{\omega}=h$ to produce the binary outputs $c_{\omega}$, which are averaged to calculate the correlation sum $\widehat{C}_{m}(h)$.

The superscript $T$ indicates that these estimators are calculated from $T_{m}$.

The correlation integral is estimated through the correlation sum $\widehat{G}_{m}(h)$. Given a scalar time series $\left\{x_{n}\right\}_{n=1}^{N}$ and the parameters $m \in \mathbb{N}$ and $\tau \in \mathbb{N}$, the $m$-dimensional vectors $\mathbf{x}_{i}=\left\{x_{i}, x_{(i+\tau)}, x_{(i+2 \tau)} \ldots, x_{[i+(m-1) \tau]}\right\}$ with $1 \leqslant i \leqslant L=N-(m-1) \tau$ must be considered. Defining the distance between embedded vectors as the Euclidean norm $\tilde{z}_{\omega}=\left\|\mathbf{x}_{\mathbf{i}}-\mathbf{x}_{\mathbf{j}}\right\|$, where $\omega=\{(i, j) / i \neq j, i=1,2, \ldots, L, j=1,2, \ldots, L\} \quad$ and $Q=L(L-1)$, the correlation sum is defined as [11]

$$
\begin{aligned}
\widehat{G}_{m}(h) & =\frac{1}{L(L-1)} \sum_{i=1}^{L} \sum_{j \neq i}^{L} g\left(\left\|\mathbf{x}_{i}-\mathbf{x}_{j}\right\| / h\right) \\
& =\frac{1}{Q} \sum_{\omega=1}^{Q} g\left(\tilde{z}_{\omega} / h\right),
\end{aligned}
$$

where $g(t)$ is the kernel function. This procedure can be translated into an algorithm called the correlation algorithm, which involves three steps. The first step is the calculation of all distances $\tilde{z}_{\omega}$ between phase-space vectors. Then for a fixed value $h$, the kernel function must be evaluated at $\tilde{z}_{\omega} / h$, and the correlation sum is obtained as the average through $\omega$. Finally, this procedure must be repeated over a range of $h$ values. When the Heaviside function is taken as a kernel [3], the aforementioned methodology can be summarized as in Fig. 1. In this special case, the algorithm compares each distance $\tilde{z}_{\omega}$ with a threshold $\theta=h$ producing a binary output $c_{\omega}$. Then the correlation sum $\widehat{C}_{m}(h)$ is computed as the average through all outputs. Basically, this procedure can be seen as a parallel array of threshold comparators similar to those modeling flash analog-to-digital converters (ADC).

It is well known that the ADC performance can be enhanced through the dithering technique, which consists in adding a random signal (commonly white Gaussian noise) to the input signal before its digitization. This is done in order to reduce the undesirable effect of quantization noise as well as to increase the dynamical range of the ADC [24-26]. The enhancement of the analog-to-digital conversion process by adding noise is called stochastic resonance [26,27]. Moreover, the stochastic resonance phenomenon displayed by the dithering technique is called suprathreshold stochastic resonance [27].
The suprathreshold stochastic resonance model consists of a parallel array of comparators or one-bit quantizers. At each quantizer, an independent and identically distributed noise realization is added to an input signal, which can be deterministic [28] or stochastic [29]. The idea is to elicit a distinct output at each quantizer in response to a common input signal. Finally, a global output is obtained as the average over all quantizer outputs [28-33]. It has been proven in [29] that injecting noise into this kind of network can improve the estimation of statistical parameters of the input signal. The enhancement relies on the fact that the added noise can elicit a better representation of the information contained in the input variable.

There is a deep similarity between the correlation algorithm and the suprathreshold stochastic resonance model. This has led us to consider the possibility that estimation of invariants such as $D$ and $K_{2}$ can be done by injecting noise into the comparators of the correlation algorithm. We will explore this idea in the next section.

\section{NOISE-ASSISTED CORRELATION INTEGRAL}

In this section, we develop the foundations of the noiseassisted correlation integral (NCI). Our departure will be the noise-assisted correlation algorithm (see Fig. 2), which is inspired by the suprathreshold stochastic resonance model. This algorithm consists of $Q$ one-bit quantizers (or comparators) with threshold value $\theta_{\omega}=0, \omega=1,2, \ldots, Q$. Each quantizer receives as input an independent and identically distributed (iid) sample $\tilde{z}_{\omega} \sim f_{\tilde{z}}$ (with support $[0, \infty)$ ) minus a sample of iid noise $\mu_{\omega}$ with a cumulative distribution function $F_{\mu}$, also independent of $\tilde{z}_{\omega}$. For a single quantizer, the output $s_{\omega}$ is ruled by

$$
s_{\omega}= \begin{cases}0 & \text { if } \tilde{z}_{\omega}-\mu_{\omega} \geqslant \theta_{\omega}, \\ 1 & \text { otherwise. }\end{cases}
$$

Finally the global output $\widehat{S}_{m}(h)$ is calculated as the average over all quantizer's outputs.

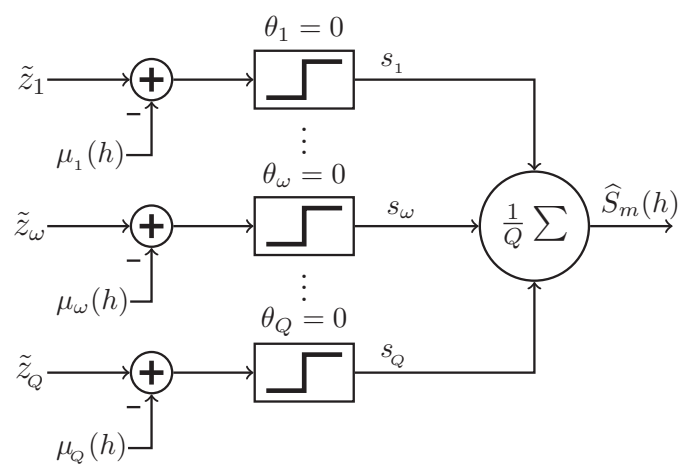

FIG. 2. Noise-assisted correlation algorithm, consisting of $Q$ quantizers with threshold $\theta_{\omega}=0$. Each quantizer receives an input that is composed of the subtraction of an iid noise realization $\mu_{\omega}$, whose PDF depends on a parameter $h$, from the distance between a pair of phase-space vectors $\tilde{z}_{\omega}$. The binary outputs of all quantizers $s_{\omega}$ are averaged to calculate the noise-assisted correlation sum $\widehat{S}_{m}(h)$. 
Based on Eq. (10), we can calculate the probability that the $\omega$ th quantizer is set to zero given the input $\tilde{z}_{\omega}$ as

$$
\begin{aligned}
\operatorname{Pr}\left\{s_{\omega}=0 \mid \tilde{z}_{\omega}\right\} & =\operatorname{Pr}\left\{\tilde{z}_{\omega}-\mu_{\omega} \geqslant \theta_{\omega}\right\} \\
& =\operatorname{Pr}\left\{\tilde{z}_{\omega}-\mu_{\omega} \geqslant 0\right\} \\
& =\operatorname{Pr}\left\{\mu_{\omega} \leqslant \tilde{z}_{\omega}\right\} \\
& =F_{\mu}\left(\tilde{z}_{\omega}\right) .
\end{aligned}
$$

We can deduce now the probability that the $\omega$ th quantizer is turned on given the input $\tilde{z}_{\omega}$ as

$$
\begin{aligned}
\operatorname{Pr}\left\{s_{\omega}=1 \mid \tilde{z}_{\omega}\right\} & =1-\operatorname{Pr}\left\{s_{\omega}=0 \mid \tilde{z}_{\omega}\right\} \\
& =1-F_{\mu}\left(\tilde{z}_{\omega}\right) .
\end{aligned}
$$

If $\widehat{S}$ is the number of quantizers turned on, the probability of $\widehat{S}=k$ given the input $\tilde{z}_{\omega}$ is given by the binomial distribution

$$
\operatorname{Pr}\left\{\widehat{S}=k \mid \tilde{z}_{\omega}\right\}=\left(\begin{array}{l}
Q \\
k
\end{array}\right)\left[1-F_{\mu}\left(\tilde{z}_{\omega}\right)\right]^{k} F_{\mu}\left(\tilde{z}_{\omega}\right)^{Q-k} .
$$

Then the marginal probability of having $k$ quantizers turned on is

$$
\operatorname{Pr}\{\widehat{S}=k\}=\int_{0}^{\infty}\left(\begin{array}{l}
Q \\
k
\end{array}\right)\left[1-F_{\mu}\left(\tilde{z}_{\omega}\right)\right]^{k} F_{\mu}\left(\tilde{z}_{\omega}\right)^{Q-k} f_{\tilde{z}}\left(\tilde{z}_{\omega}\right) d \tilde{z}_{\omega},
$$

and the expected value of $\widehat{S}$ is

$$
\langle\widehat{S}\rangle=Q \int_{0}^{\infty}\left[1-F_{\mu}\left(\tilde{z}_{\omega}\right)\right] f_{\tilde{z}}\left(\tilde{z}_{\omega}\right) d \tilde{z}_{\omega} .
$$

Defining the estimator $S_{m}(h)=\langle\widehat{S}\rangle / Q$, making $\tilde{z}=\tilde{z}_{\omega}$, and assuming that the noise distribution depends on a parameter $h$, i.e., $F_{\mu}(t ; h)$, we can write from the last equation

$$
\begin{aligned}
S_{m}(h) & =\langle\widehat{S}\rangle / Q \\
& =\int_{0}^{\infty}\left[1-F_{\mu}(\tilde{z} ; h)\right] f_{\tilde{z}}(\tilde{z}) d \tilde{z} \\
& =\int_{0}^{\infty} \widehat{F}_{\mu}(\tilde{z} ; h) f_{\tilde{z}}(\tilde{z}) d \tilde{z},
\end{aligned}
$$

where $\widehat{F}_{\mu}(t ; h)$ is the complementary cumulative distribution function of the noise $\mu_{\omega}$. We will call $S_{m}(h)$ the noise-assisted correlation integral. It can be observed that Eq. (11) is similar to Eq. (1) but now the kernel function is $g(\tilde{z} ; h)=\widehat{F}_{\mu}(\tilde{z} ; h)$. It is clear that all complementary cumulative distribution functions decrease monotonically, meeting the first condition that allows the scaling behavior of the correlation integral [Eq. (4)]. The second condition can be met by using distributions with compact support or distributions whose complementary cumulative distribution functions decay faster or equal to the complementary cumulative distribution function of the exponential distribution.

As particular examples, we can show that if the added noise $\mu_{\omega}$ follows a deterministic distribution with probability mass function $f_{\mu}(\tilde{z} ; h)=\delta[\tilde{z} / h-1]$ and cumulative distribution function $F_{\mu}(\tilde{z} ; h)=H(\tilde{z} / h-1)$, then Eq. (11) can be written as

$$
\begin{aligned}
S_{m}(h) & =\int_{0}^{\infty}[1-H(\tilde{z} / h-1)] f_{\tilde{z}}(\tilde{z}) d \tilde{z} \\
& =\int_{0}^{\infty} H(1-\tilde{z} / h) f_{\tilde{z}}(\tilde{z}) d \tilde{z} \\
& =C_{m}(h),
\end{aligned}
$$

which is the standard correlation integral [see Eq. (2)]. On the other hand, the GCI can be derived from Eq. (11) by adding noise with Rayleigh distribution $\mu_{\omega} \sim R(\tilde{z} ; \sqrt{2} h)$. This is the distribution of the Euclidean distance between two iid bidimensional Gaussian random vectors of zero mean and variance $2 h^{2}$. Given that its complementary cumulative distribution function is $\widehat{F}_{\mu}(\tilde{z} ; \sqrt{2} h)=e^{-\tilde{z}^{2} / 4 h^{2}}$, we can write

$$
\begin{aligned}
S_{m}(h) & =\int_{0}^{\infty} e^{-\tilde{z}^{2} / 4 h^{2}} f_{\tilde{z}}(\tilde{z}) d \tilde{z} \\
& =T_{m}(h) .
\end{aligned}
$$

These two examples demonstrate that $C_{m}(h)$ and $T_{m}(h)$ can be thought of as particular cases of $S_{m}(h)$, which means that they can be estimated by adding noise, as was described earlier. It is important to mention that the main difference between the standard correlation algorithm and the noise-assisted algorithm proposed herein lies in the nature of the threshold used to count the number of neighbor phase-space vectors. The former uses a deterministic threshold (see Fig. 1). On the other hand, the addition of noise $\mu_{\omega}$ in the latter is equivalent to thresholding each distance $\tilde{z}_{\omega}$ with a stochastic threshold that follows the same distribution of $\mu_{\omega}$.

To calculate the noise-assisted correlation sum, Algorithm 1 should be followed.

Algorithm 1 Noise-assisted correlation algorithm.

1: Form $m$-dimensional phase-space vectors from the temporal series and calculate all pairwise squared distances

$z_{\omega}$ with $1 \leqslant \omega \leqslant Q=L(L-1)$.

2: Fix the value of the parameter $h$ and obtain $Q$ realization of noise $\mu_{\omega}$ from the distribution $f_{\mu}(\mu ; h)$.

3: Calculate the binary variable:

$$
s_{\omega}(h)= \begin{cases}0 & \text { if } z_{\omega}-\mu_{\omega} \geqslant 0, \\ 1 & \text { otherwise. }\end{cases}
$$

4: Calculate the noise-assisted correlation sum as

$$
\widehat{S}_{m}(h)=\frac{1}{Q} \sum_{\omega=1}^{Q} s_{\omega}(h) \text {. }
$$

5: Repeat steps 1-4 for all values of $h$ and $m$.

One advantage of the noise-assisted algorithm is related to the evaluation of kernel functions. As mentioned before, the correlation integral is estimated using the correlation sum, which requires many evaluations of the chosen kernel function. For kernel functions that require numerical integration for their evaluation, this methodology becomes computationally expensive. However, if this function can be written as the complementary cumulative distribution functions of a random variable, the estimation of the correlation sum can be done using this new approach.

Another interesting fact about the NCI is that the GCI can be estimated using noise with Rayleigh distribution, which is a chi distribution $\left(\chi_{\beta}\right)$ with two degrees of freedom $(\beta=2)$. A $\chi_{\beta}$ 
with $\beta$ degrees of freedom is the distribution of the Euclidean distance between two $\beta$-dimensional iid random vectors drawn from a multivariate normal distribution. This thought led us to hypothesize that it may be possible to introduce a correlation integral using a new type of kernel function that can generalize the GCI.

\section{THE $\boldsymbol{U}$-CORRELATION INTEGRAL}

A particular case of the NCI occurs from a generalization of the GCI. As we mentioned before, the noise-assisted correlation algorithm is characterized by the addition of random noise $\mu_{\omega}$ that induces a stochastic threshold with the same distribution.

In Eq. (12), we showed that the GCI can be induced by adding noise with Rayleigh distribution. Then, the estimation of the GCI for different $m$ values involves distances whose PDF is changing with $m$ and thresholds with a distribution that does not. However, it is natural to think that the distribution of the threshold should also be a function of the embedding dimension.

In this sense, we need to think of a kernel function that can take into account information of the embedding dimension. That kernel function is

$$
g(z ; h)=\frac{\Gamma\left(\beta / 2, z / h^{2}\right)}{\Gamma(\beta / 2)},
$$

where $\Gamma(a, t)$ is the upper incomplete Gamma function, $\Gamma(a)$ is the Gamma function, and $z=\tilde{z}^{2}$ is the squared distance between phase-space vectors. This kernel can be induced by the addition of noise $\mu_{\omega}$ that follows a chi-squared distribution $\left(\chi_{\beta}^{2}\right)$ with variance $h^{2} \cdot \chi_{\beta}^{2}$ is the distribution of the squared Euclidean distance between two $\beta$-dimensional random vectors taken from a $\beta$-dimensional normal distribution. Then, the $\beta$ parameter can be used to incorporate information of the embedding dimension.

Note that we are using the $\chi_{\beta}^{2}$ instead of the $\chi_{\beta}$. This is because it is more expensive, computationally speaking, to calculate the distances between $m$-dimensional vectors than to calculate their squared distances. Moreover, it is faster to generate noise $\mu_{\omega}$ with $\chi_{\beta}^{2}$ than with $\chi_{\beta}$. As a consequence, from now on we will work with the squared distance $z=\tilde{z}^{2}$. This also implies that the inputs to the noiseassisted correlation algorithm must be the squared distances $z_{\omega}=\tilde{z}_{\omega}^{2}$.

To deduce the $U$-correlation integral (UCI), we also need an expression for the distribution of the squared distance between phase-space vectors $f_{z}$. Oltmans et al. derived an equation for the distribution of the squared distance between $m$-dimensional phase-space vectors contaminated with iid normal noise with zero mean and variance $\sigma^{2}$ [see Eq. (18) in Ref. [34]]:

$f_{z}(m, \sigma ; z)=\frac{z^{m / 2-1}}{2(2 \sigma)^{m-D}} \frac{\Gamma(D / 2)}{\Gamma(m / 2)}{ }_{1} F_{1}\left(\frac{m-D}{2}, \frac{m}{2} ;-\frac{z}{4 \sigma^{2}}\right)$,

where $F_{1}(a, b ; t)$ is Kummer's confluent hypergeometric function. This distribution arises under the assumption that the PDF of the unperturbed pairwise distances between phase-space vectors behaves as $h^{D-1}$ for a certain range of $h$ values [34]. If each of the coordinates of these vectors is perturbed with uncorrelated Gaussian noise of variance $\sigma^{2}$, then the PDF of the noisy distances will follow Eq. (14). This was demonstrated in [34] through a statistical approach in which Kummer's confluent hypergeometric function results from the convolution of the PDF of the unperturbed distances and the Gaussian distribution.

Substituting Eqs. (13) and (14) into Eq. (11), we define the $U$-correlation integral as

$$
\begin{aligned}
U_{m}^{\beta}(h)= & \int_{0}^{\infty} \frac{\Gamma\left(\beta / 2, z / h^{2}\right)}{\Gamma(\beta / 2)} \frac{z^{m / 2-1}}{2(2 \sigma)^{m-D}} \frac{\Gamma(D / 2)}{\Gamma(m / 2)} \\
& \times{ }_{1} F_{1}\left(\frac{m-D}{2}, \frac{m}{2} ;-\frac{z}{4 \sigma^{2}}\right) d z,
\end{aligned}
$$

and it can be shown that

$$
\begin{aligned}
U_{m}^{\beta}(h)= & \frac{\widehat{\phi}}{2}\left(4 \sigma^{2}\right)^{D / 2} e^{-m \tau K_{2}} \frac{\Gamma(D / 2) \Gamma((\beta+m) / 2)}{\Gamma(\beta / 2) \Gamma(m / 2+1)}\left(\frac{h^{2}}{4 \sigma^{2}}\right)^{m / 2} \\
& \times{ }_{2} F_{1}\left(\frac{\beta+m}{2}, \frac{m-D}{2} ; \frac{m+2}{2} ;-\frac{h^{2}}{4 \sigma^{2}}\right),
\end{aligned}
$$

where ${ }_{2} F_{1}(a, b ; c ; t)$ is the Gauss hypergeometric function and $\widehat{\phi}$ is a normalization constant. This CI introduces a new parameter $\beta$, which is the degree of freedom of the noise $\mu_{\omega}$ used in the noise-assisted correlation algorithm. Since the $\chi_{\beta}^{2}$ can be thought of as the distribution of the distance between two $\beta$-dimensional normally distributed random vectors, we will refer to $\beta$ as the noise dimension.

\section{A. $\mathbf{U C I} I^{\beta=2}$}

As we mentioned before, the GCI is a particular case of the UCI. This can be verified by setting $\beta=2$ in Eq. (15):

$$
\begin{aligned}
U_{m}^{\beta=2}(h)= & \frac{\widehat{\phi}}{2}\left(4 \sigma^{2}\right)^{D / 2} \Gamma(D / 2) e^{-m \tau K_{2}}\left(\frac{h^{2}}{4 \sigma^{2}}\right)^{m / 2} \\
& \times{ }_{2} F_{1}\left(\frac{m+2}{2}, \frac{m-D}{2} ; \frac{m+2}{2} ;-\frac{h^{2}}{4 \sigma^{2}}\right) .
\end{aligned}
$$

Taking into account that ${ }_{2} F_{1}(a, b ; a ; t)=(1-t)^{-b}$, we have

$$
U_{m}^{\beta=2}(h)=\widehat{\phi} 2^{D-1} \Gamma(D / 2) h^{m}\left(h^{2}+4 \sigma^{2}\right)^{\frac{D-m}{2}} e^{-m \tau K_{2}} .
$$

Using Eq. (5), we can conclude that

$$
U_{m}^{\beta=2}(h)=\frac{\widehat{\phi}}{\phi} 2^{(2 D-1)} \Gamma(D / 2) T_{m}(h / 2) .
$$

This result shows us that the $U$-correlation integral with $\beta=$ 2 is just a scaled version of the Gaussian kernel correlation integral. This means that they describe the same $h$ dependence.

To numerically validate the aforementioned results, we perform a set of simulations using time series coming from the Henon map:

$$
x_{n+1}=1-a x_{n}^{2}+b x_{n-1},
$$

with $a=4$ and $b=0.3$. We must clarify that we have chosen this map and the Mackey-Glass system [Eq. (26) will be used in a further simulation] because they have been widely 


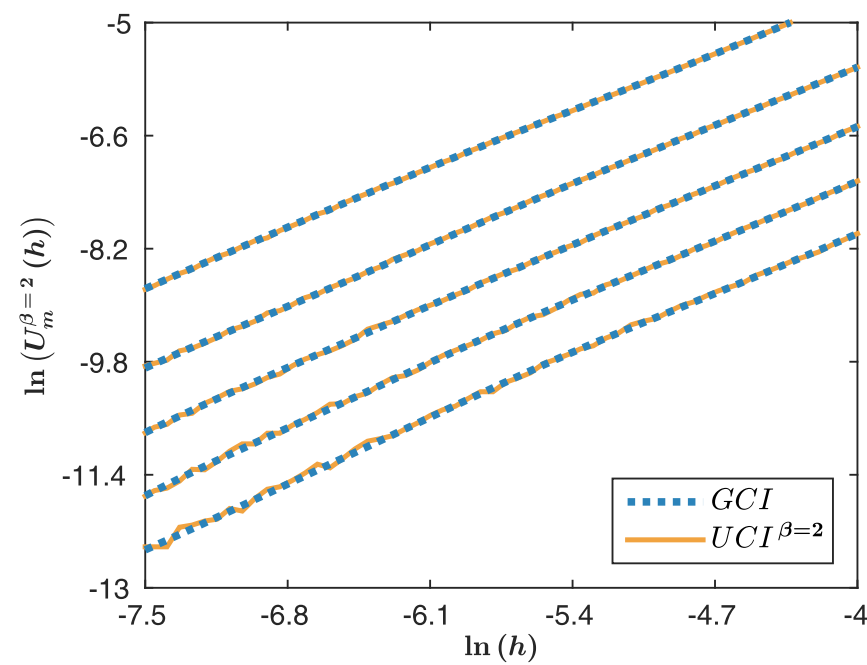

FIG. 3. Noiseless Henon map. Log-log plot of GCI (dotted blue line) and $\mathrm{UCI}^{\beta=2}$ (solid orange line) with $m=\{2,4,6,8,10\}(m=2$ top and $m=10$ bottom).

used in the literature related to the estimation of invariants from time series. In this way, these invariants have been well characterized, and the results can be easily compared with similar studies already published by other authors [4,20-23,34-36].

Figure 3 shows a log-log plot of the $U_{m}^{\beta=2}(h)\left(\mathrm{UCI}^{\beta=2}\right)$ and the $T_{m}(h / 2)$. This example was calculated from a single realization of the Henon map in the absence of noise. The number of embedding vectors was kept constant at $Q=5000$ through the different values of $m=\{2,4, \ldots, 10\}$, and the embedding lag was $\tau=1$. As can be seen, for small values of $h$, the $\mathrm{UCI}^{\beta=2}$ fluctuates slightly around the value of the GCI. However, for larger $h$ values, both correlation integrals are very similar. Notice that the slopes of all of the curves are equal, which means that both correlation integrals approach the same value of $D$. This can be observed in Fig. 4, which shows the reported value of the correlation dimension for the Henon map, $D=1.22[11,16]$, the logarithmic derivative of the $\mathrm{UCI}^{\beta=2}$, and the logarithmic derivative of the GCI as functions of $\ln h$. It can be observed how both the $\mathrm{UCI}^{\beta=2}$ and the GCI oscillate around this reported value of $D$.

The fluctuation of the $\mathrm{UCI}^{\beta=2}$ is associated with statistical instabilities as a consequence of the finite number of available phase-space vectors. This oscillation can be reduced by increasing the number of comparators $Q$ in the noise-assisted correlation algorithm. It can be done in two different ways. The first one is to increase the time-series length $N$. The second one is to create copies of each squared distance $z_{\omega}$, which will increase the number of comparators. Then a different realization of noise $\mu_{\omega}$ must be added to each copy, and the thresholding procedure must be applied. Finally, the correlation sum $\widehat{S}(h)$ is obtained as the average over all comparator outputs. As a technical detail, we must mention that all the derivatives in this document were approximated using a wavelet transform approach [37].

These results lead us to conclude that the $U$-correlation integral can be considered as a generalization of the GCI. We

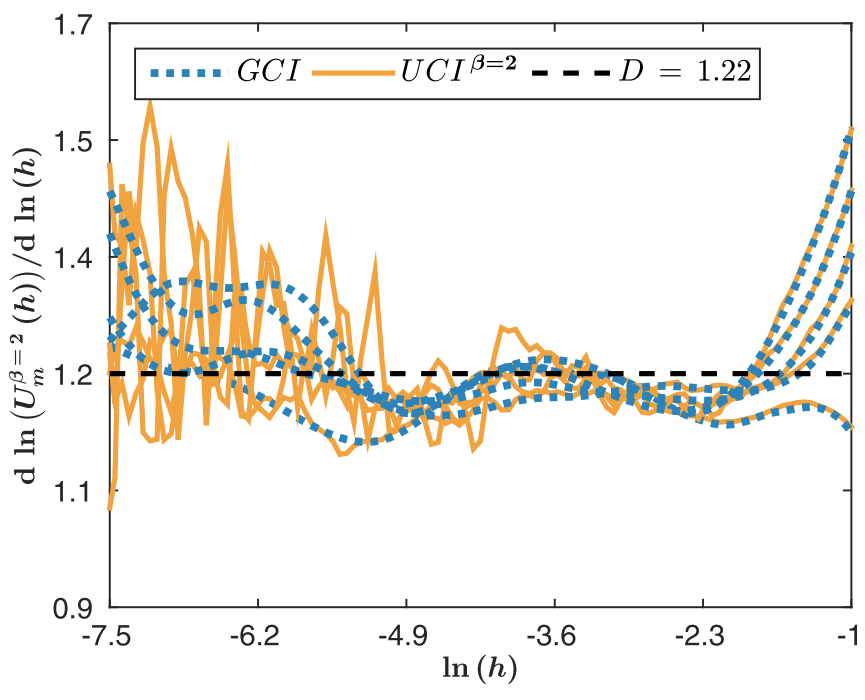

FIG. 4. Estimation of the correlation dimension for the noiseless Henon map using $m=\{2,4,6,8,10\}$. Log-derivatives of GCI (dotted blue line) and $\mathrm{UCI}^{\beta=2}$ (solid orange line). The reported value of correlation dimension $D=1.22$ (dashed black line).

found that, computationally speaking, it is faster to calculate the GCI than the $\mathrm{UCI}^{\beta=2}$, since for a fixed value of $h$ the evaluation of the function $e^{-z^{2} / 4 h^{2}}$ is faster than the generation of noisy samples with $\chi_{\beta=2}^{2}(h)$. However, for values of $\beta \geqslant 3$ the evaluation of the incomplete $\Gamma$ function will require numerical integration, and this can be slower than generating samples of $\chi_{\beta}^{2}(h)$ noise.

\section{B. $\mathbf{U C I}^{\beta=m}$}

The kernel function proposed in Eq. (13) introduces a new parameter $\beta$ (we will refer to $\beta$ as the noise dimension), which describes the degrees of freedom of the $\chi_{\beta}^{2}$ distributed noise used in the noise-assisted correlation algorithm. The idea is that $\beta$ allows us to introduce information about the embedding dimension $m$ in the kernel function. This will be useful for the estimation of the system's invariants.

To illustrate this idea, we will set the noise dimension equal to the embedding dimension $(\beta=m)$ in Eq. (15) and define the correlation integral $U_{m}^{\beta=m}(h)$ as

$$
\begin{aligned}
U_{m}^{\beta=m}(h)= & \frac{\widehat{\phi}}{2}\left(4 \sigma^{2}\right)^{D / 2} e^{-m \tau K_{2}} \frac{\Gamma(D / 2) \Gamma(m)}{\Gamma(m / 2) \Gamma(m / 2+1)} \\
& \times\left(\frac{h^{2}}{4 \sigma^{2}}\right)^{m / 2}{ }_{2} F_{1}\left(m, \frac{m-D}{2} ; \frac{m+2}{2} ;-\frac{h^{2}}{4 \sigma^{2}}\right),
\end{aligned}
$$

from which we can propose a new correlation dimension functional (see Appendix A):

$$
\begin{aligned}
\frac{d \ln U_{m}^{\beta=m}(h)}{d \ln h}= & m-\frac{2 m(m-D)}{m+2}\left(\frac{h^{2}}{4 \sigma^{2}}\right) \\
& \times \frac{{ }_{2} F_{1}\left(m+1, \frac{m-D}{2}+1 ; \frac{m+2}{2}+1 ; \frac{h^{2}}{4 \sigma^{2}}\right)}{{ }_{2} F_{1}\left(m, \frac{m-D}{2} ; \frac{m+2}{2} ; \frac{-h^{2}}{4 \sigma^{2}}\right)} .
\end{aligned}
$$




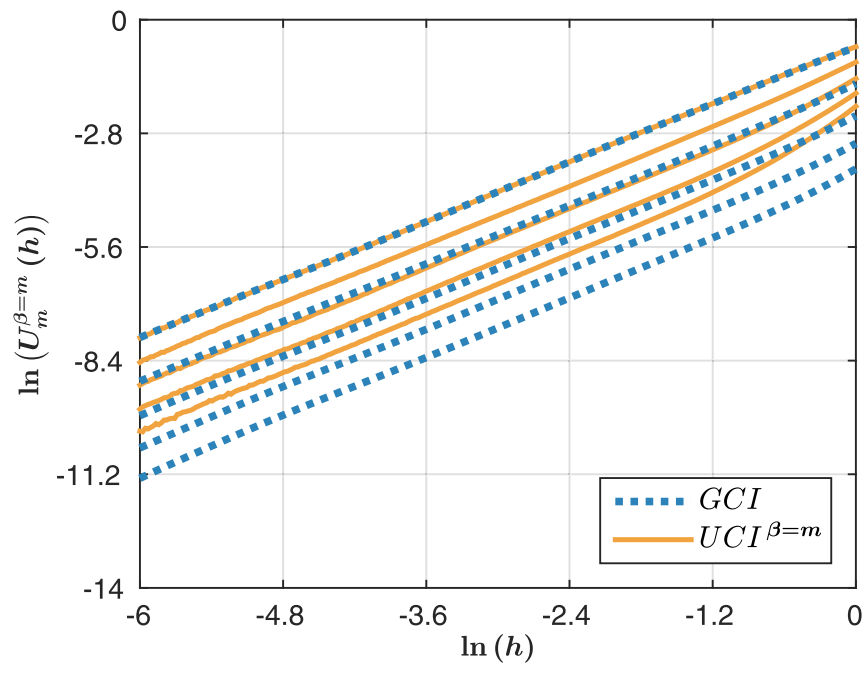

FIG. 5. Noiseless Henon map. Log-log plot of the GCI (dotted blue line) and $\mathrm{UCI}^{\beta=m}$ (solid orange line) with $m=\{2,4,6,8,10\}$ ( $m=2$ top and $m=10$ bottom).

It can be assessed that this functional approaches $D$ when $\sigma \ll h$ and $m$ when $\sigma \gg h$ (see Appendix A). This behavior is similar to the correlation dimension functional based on the GCI $[11,13]$. Figure 5 shows a $\log -\log$ plot of the $U_{m}^{\beta=m}(h)$ $\left(\mathrm{UCI}^{\beta=m}\right)$ and the GCI calculated with different values of $m=$ $\{2,4, \ldots, 10\}$ for the noiseless Henon map. The number of embedding vectors was kept constant $(Q=5000)$ through the values of $m$, and the embedding lag was $\tau=1$. It can be seen that for $m=2$ (at the top), the curve corresponding to the $\mathrm{UCI}^{\beta=m}$ and the one corresponding to the GCI overlap. Moreover, the slopes of all of the curves are similar for both $\mathrm{UCI}^{\beta=m}$ and GCI, which means that both will converge to similar values of $D$. This is clear in Fig. 6, which shows the reported value of the correlation dimension for the Henon map

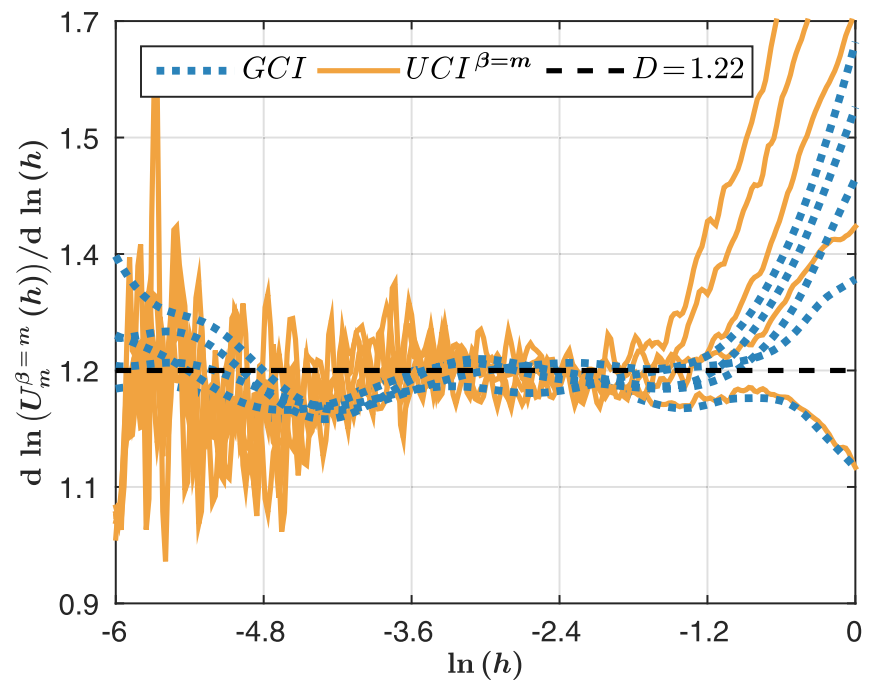

FIG. 6. Estimation of the correlation dimension for the noiseless Henon map using $m=\{2,4,6,8,10\}$. Log-derivatives of GCI (dotted blue line) and $\mathrm{UCI}^{\beta=m}$ (solid orange line). The reported value of correlation dimension $D=1.22$ (dashed black line).

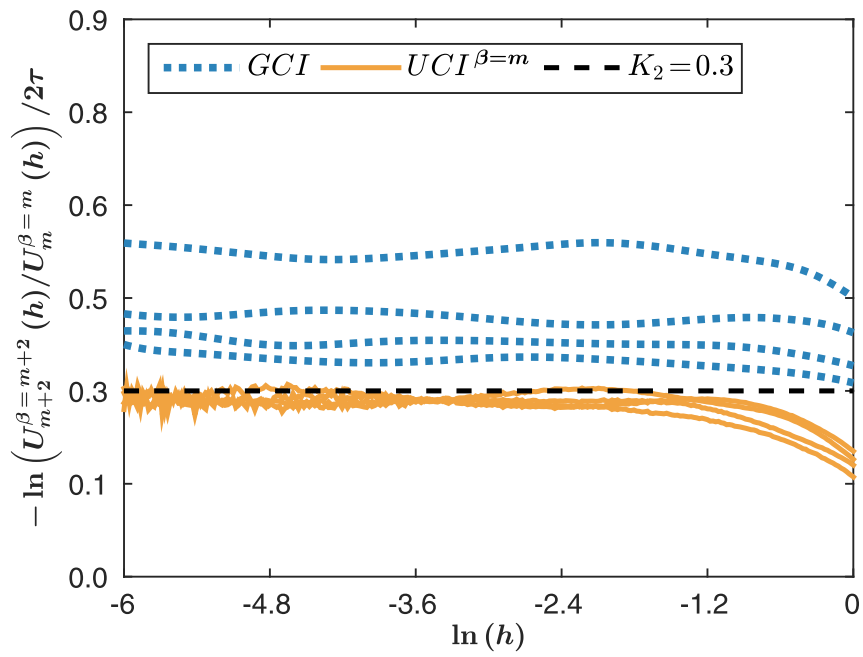

FIG. 7. Estimation of $K_{2}$ for the noiseless Henon map: Estimation based on GCI (dotted blue line), and estimation based on $\mathrm{UCI}^{\beta=m}$ (solid orange line) for $m=\{2,4,6,8\}$. The reported value of correlation entropy $K=0.3$ (dashed black line).

$D=1.22$, the logarithmic derivative of the $\mathrm{UCI}^{\beta=m}$, and the logarithmic derivative of the GCI as functions of $\ln h$. Both correlation integrals, $\mathrm{UCI}^{\beta=m}$ and GCI, approach the reported value of $D$, meaning that both estimators describe the same $h$ dependence.

One of the main differences between the $\mathrm{UCI}^{\beta=m}$ and the GCI lies in the distance between curves for different $m$ values. As is shown in Fig. 5, the difference between curves for different $m$ values is more uniform for the $\mathrm{UCI}^{\beta=m}$ than for the GCI. Since the estimation of $K_{2}$ is based on calculations of the correlation integral for different $m$ values, the last result suggests that the use of $\mathrm{UCI}^{\beta=m}$ could have an advantage over the use of GCI. We define from Eq. (17) a new entropy

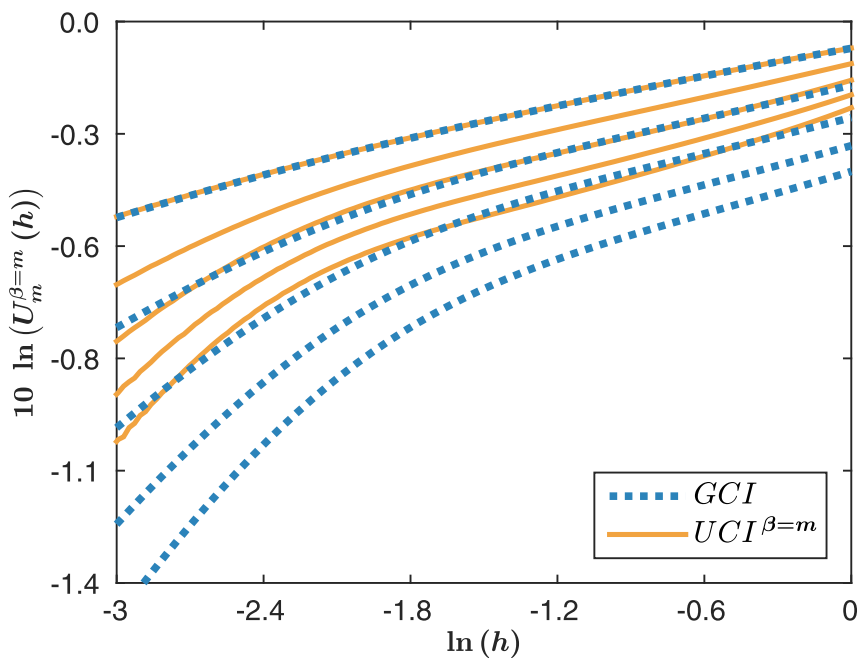

FIG. 8. Noisy Henon map $(\sigma=0.1) \log$-log plot for $m=\{2,4,6,8,10\}:$ GCI (dotted blue line) and $\mathrm{UCI}^{\beta=m}$ (solid orange line). 


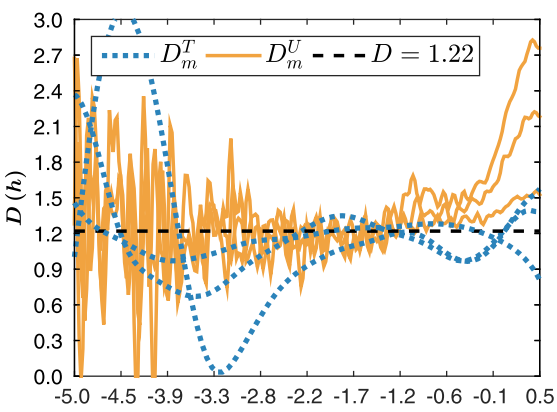

(a)

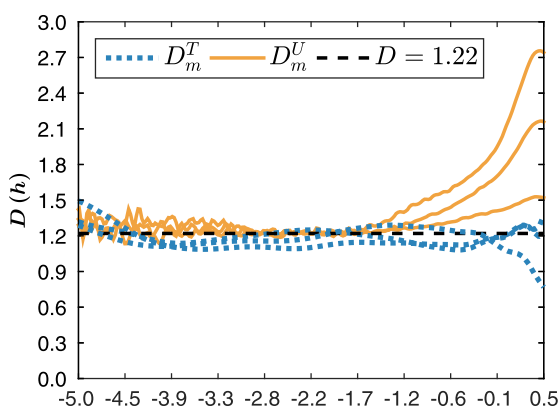

(d)

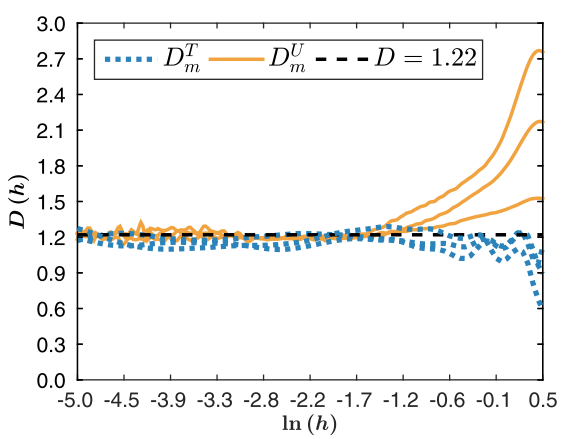

(g)

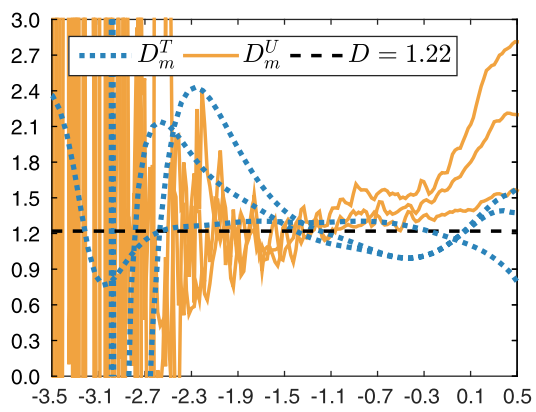

(b)

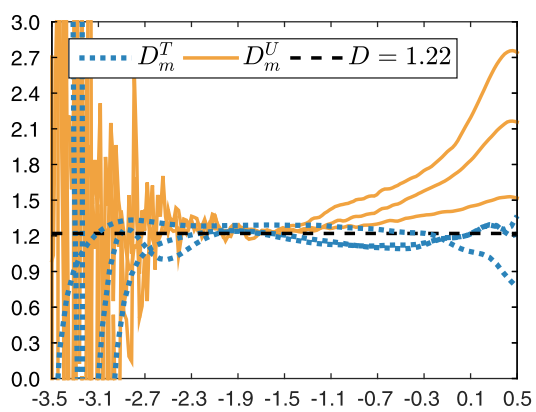

(e)

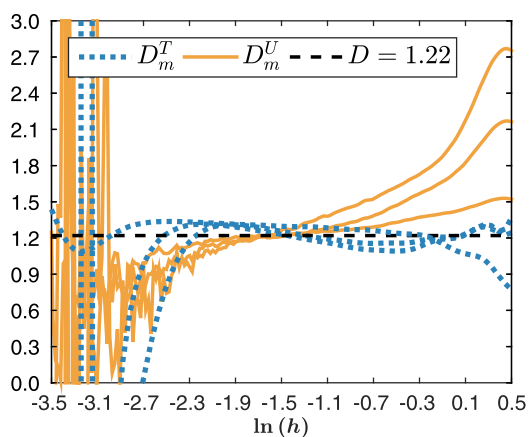

(h)

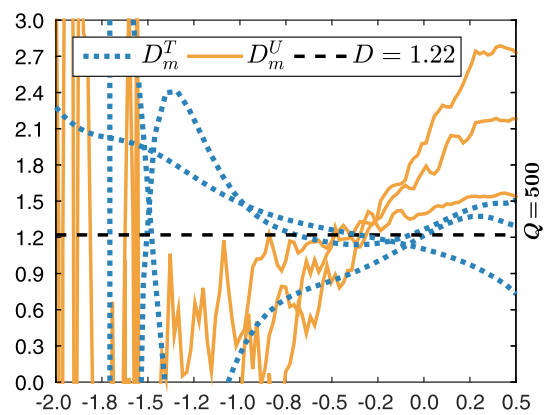

(c)

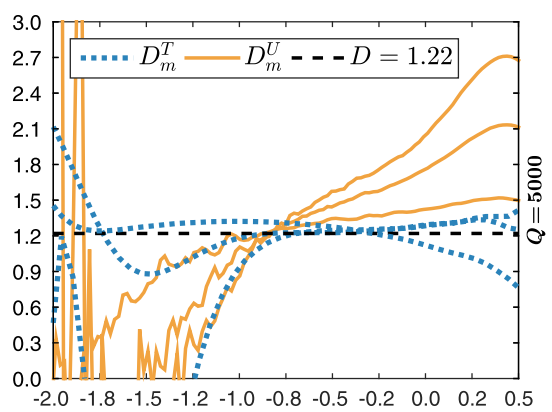

(f)

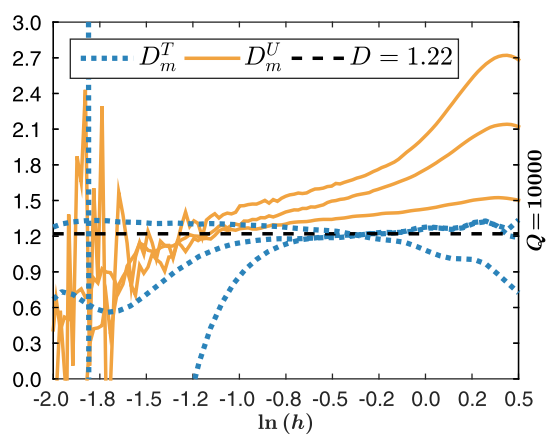

(i)

FIG. 9. Correlation dimension coarse-grained estimators for the Henon map. $D_{m}^{T}$ (dotted blue line) and $D_{m}^{U}$ (solid orange line) for $m=[4,6,8]$ and $Q=\{500,5000,10000\}$. Top row: $Q=500$ (a) noiseless, (b) $\sigma=0.05$, and (c) $\sigma=0.2$. Middle row: $Q=5000$ (d) noiseless, (e) $\sigma=0.05$, and (f) $\sigma=0.2$. Bottom row: $Q=10000$ (g) noiseless, (h) $\sigma=0.05$, and (i) $\sigma=0.2$. The reported correlation dimension value $D=1.22$ is shown by a dashed black line.

functional based on the $\mathrm{UCI}^{\beta=m}$ as

$$
\begin{aligned}
\ln \frac{U_{m+2}^{\beta=m+2}(h)}{U_{m}^{\beta=m}(h)} \\
=\ln \left\{\frac{m+1}{m+2}\right\}+\ln \frac{h^{2}}{\sigma^{2}}-2 \tau K_{2} \\
\quad+\ln \left\{\frac{{ }_{2} F_{1}\left(m+2, \frac{m-D}{2}+1 ; \frac{m+2}{2}+1 ; \frac{-h^{2}}{4 \sigma^{2}}\right)}{{ }_{2} F_{1}\left(m, \frac{m-D}{2} ; \frac{m+2}{2} ; \frac{-h^{2}}{4 \sigma^{2}}\right)}\right\} .
\end{aligned}
$$

When $\sigma \ll h$, this estimator approaches

$$
\ln \frac{U_{m+2}^{\beta=m+2}(h)}{U_{m}^{\beta=m}(h)}=-2 \tau K_{2}+\ln \left(\frac{D}{m}+1\right),
$$

and for $m$ values much bigger than $D$ it can be seen that $\ln \left[U_{m+2}^{\beta=m+2}(h) / U_{m}^{\beta=m}(h)\right] \approx-2 \tau K_{2}$.

Figure 7 shows the entropy functional based on $\mathrm{UCI}^{\beta=m}$ for $m=\{2,4,6,8\}$. It can be observed how this entropy functional converges to the reported value of correlation entropy $\left(K_{2}=\right.$ $0.3[11,16])$ faster than the estimator based on GCI $\left(K_{2} \approx\right.$ $\left.-1 / \tau \ln \left[T_{m+1}(h) / T_{m}(h)\right]\right)$.

At this point, we have proposed a correlation integral $\mathrm{UCI}^{\beta=m}$ from which we have derived a dimension and an entropy functional. We have shown that these estimators approach the reported values of dimension and entropy when the time series are clean. On the other hand, when the noise level $(\sigma)$ is increased, both the $\mathrm{UCI}^{\beta=m}$ and the GCI deviate from the power-law behavior. We must clarify that all time series used in this article were rescaled to have unitary 


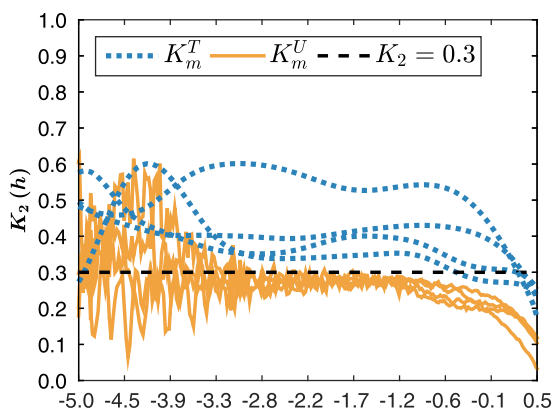

(a)

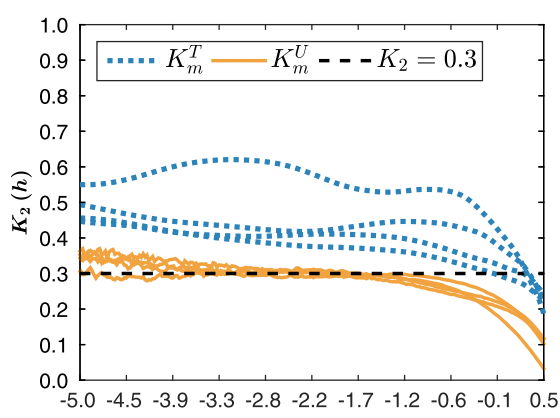

(d)

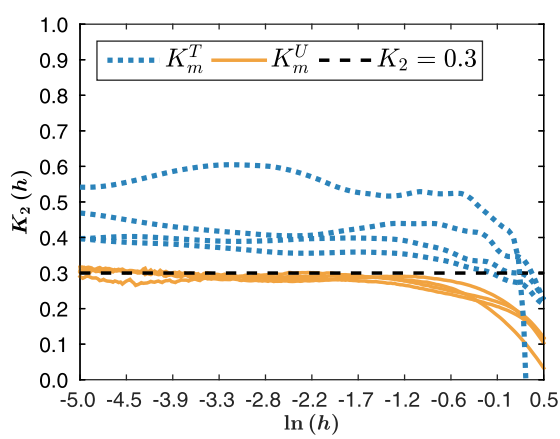

(g)

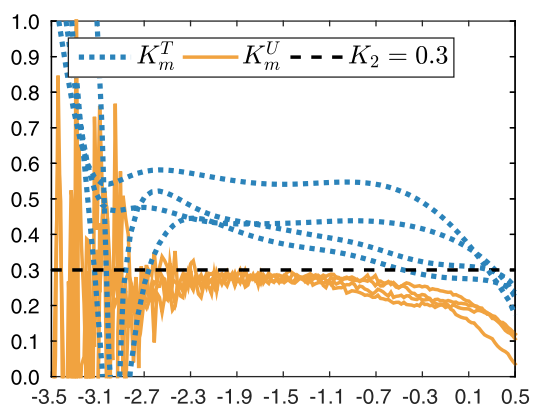

(b)

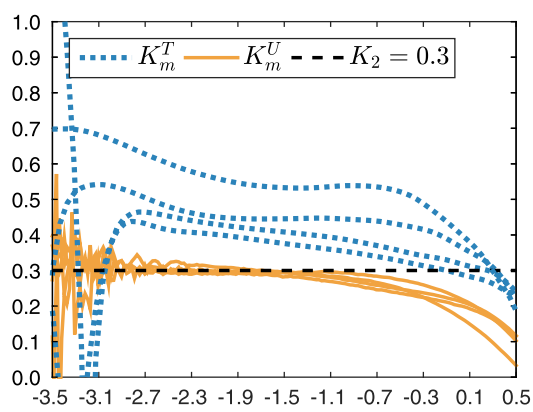

(e)

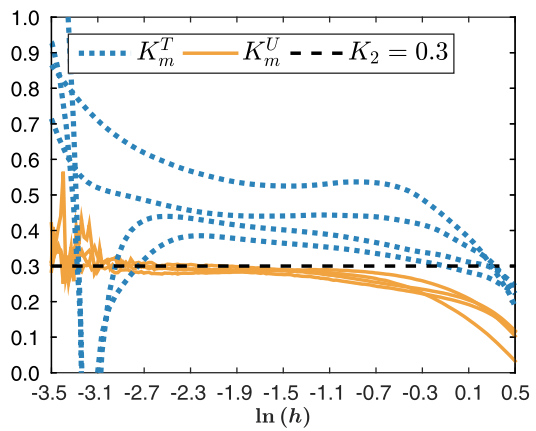

(h)

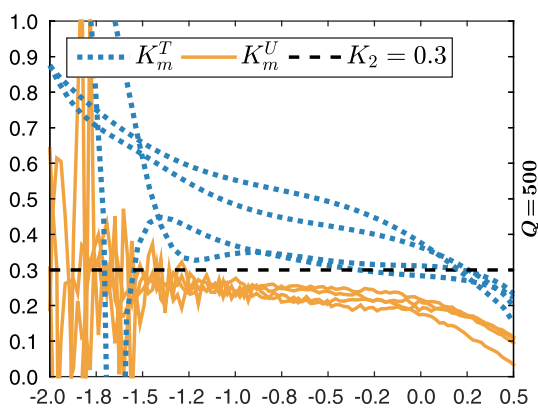

(c)

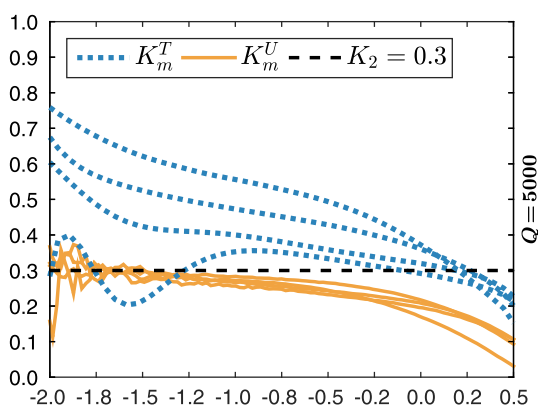

(f)

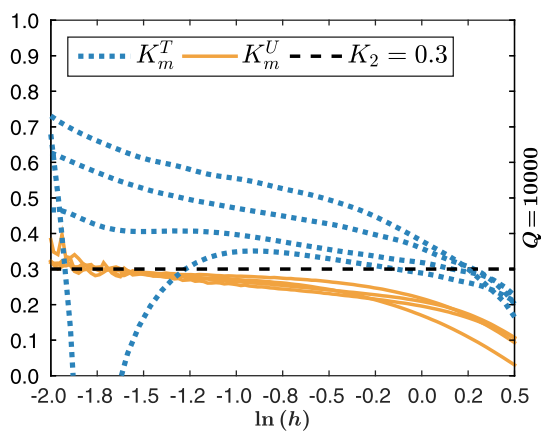

(i)

FIG. 10. Correlation entropy coarse-grained estimators for the Henon map. $K_{m}^{T}$ (dotted blue line) and $K_{m}^{U}$ (solid orange line) for $m=[2,4,6,8]$ and $Q=\{500,5000,10000\}$. Top row: $Q=500$ (a) noiseless, (b) $\sigma=0.05$, and (c) $\sigma=0.2$. Middle row: $Q=5000$ (d) noiseless, (e) $\sigma=0.05$, and (f) $\sigma=0.2$. Bottom row: $Q=10000$ (g) noiseless, (h) $\sigma=0.05$, and (i) $\sigma=0.2$. The reported correlation entropy value $K_{2}=0.3$ is shown by a dashed black line.

standard deviation. As a consequence, $\sigma$ is the noise level after rescaling, i.e.,

$$
\sigma=\frac{\sigma_{n}}{\sqrt{\sigma_{c}^{2}+\sigma_{n}^{2}}},
$$

where $\sigma_{n}^{2}$ is the noise variance and $\sigma_{c}^{2}$ is the variance of the clean time series. In this sense, $\sigma=0$ corresponds to clean time series and $\sigma=1$ implies only noise.

Figure 8 shows that for $\sigma=0.1$, both $\mathrm{UCI}^{\beta=m}$ and GCI bend off to a larger slope when $h$ is small. It can be seen that for large $h$ values, the differences between curves for consecutive $m$ values are more consistent for the $\mathrm{UCI}^{\beta=m}$ than for the GCI.

Since there are no practical applications free of some noise, it is important to find analytic expressions that allow us to estimate $D, K_{2}$, and $\sigma$ from functions that depend on $h$ and $m$. In the next section, we will present coarse-grained estimators that will be tested under different conditions.

\section{COARSE-GRAINED ESTIMATORS BASED ON THE UCI ${ }^{\beta=m}$}

The coarse-grained estimators allow us to visually inspect the data for proper scaling regions as well as to estimate the invariants $D, K_{2}$, and $\sigma$. To derive these estimators, we will take advantage of some identities of the Gauss hypergeometric function and the $\beta$ parameter. We will compare our coarse-grained estimators with those based on the GCI [19], given by Eqs. (6), (7), and (8). These estimators can be efficiently computed and, more importantly, their calculation only depends on the estimation of correlation integrals for 
consecutive embedding dimensions, i.e., no other external quantities or parameters are needed.

\section{A. Correlation dimension $(D)$}

It is shown in Appendix B that a coarse-grained dimension estimator in terms of $\mathrm{UCI}^{\beta=m}$ can be defined as

$$
\begin{aligned}
D_{m}^{U}(h)= & \left(\frac{4 \sigma^{2}+h^{2}}{h^{2}}\right) \frac{d \ln U_{m}^{\beta=m}(h)}{d \ln h} \\
& +\frac{4 \sigma^{2}}{h^{2}}(2 m-2)\left[\frac{U_{m}^{\beta=m-2}(h)}{U_{m}^{\beta=m}(h)}-1\right],
\end{aligned}
$$

where $U_{m}^{\beta=m}(h)$ is the UCI calculated by adding noise with the same dimension as the embedding vectors, i.e., $\beta=m$. The estimator $U_{m}^{\beta=m-2}(h)$ is the UCI calculated using embedding dimension $m$ and noise dimension $\beta=m-2$. The superscript $U$ denotes that this estimator comes from the UCI. It can be seen that for $\sigma \rightarrow 0$, this estimator approaches $d \ln U_{m}^{\beta=m}(h) / d \ln h=D$ (see Appendix A). This behavior has been observed in other coarse-grained estimators, such as the ones proposed by Diks [13], Nolte et al. [19], and Jayawardena et al. [22].

Figure 9 shows the behaviors of $D_{m}^{U}$ and $D_{m}^{T}$ as functions of $h$ for $m=\{4,6,8\}$. These estimators were calculated from time series coming from the Henon map with different noise levels (noiseless, $\sigma=0.05$, and $\sigma=0.2$ ) and data lengths, reflected in the number of available phase-space vectors $Q=\{500,5000,10000\}$. The reported value of the correlation dimension $D=1.22$ is also shown.

For the noiseless case, we can see from the first column of Fig. 9 how for a range of $\ln h$ values the estimator $D_{m}^{U}$ oscillates around the reported value of $D$, regardless of $m$. Moreover, as the number of available phase-space vectors increases, this oscillation is attenuated. This result is very similar to the one achieved with the estimator $D_{m}^{T}$.

It can be observed in Figs. 9(b), 9(e), and 9(h) that for a low noise level $(\sigma=0.05)$, the scaling range is shorter than in the noiseless case. However, for a large number of phase-space vectors, there still can be found a scaling region. There is something interesting about the estimator $D_{m}^{U}$ in the presence of noise that we have observed through a large number of simulations. From Fig. 9(b) it can be seen that $D_{m}^{U}$ approaches the reported value of $D$ when $\ln h \approx-1.3$. At this value, the curves for different embedding dimensions $m$ get the closest. In other words, the $D_{m}^{U}$ approaches the reported value of $D$ at the $h$ value corresponding to the minimum of the variance of $D_{m}^{U}$ calculated through the different $m$ values. On the other hand, for values of $Q=\{5000,10000\}$ it is shown in Figs. 9(e) and $9(\mathrm{~h})$ that a scaling region can be recognized $(-1.9 \leqslant$ $\ln h \leqslant-1.3)$.

For high noise levels $(\sigma=0.2)$, the third column of Fig. 9 shows that it is difficult to find a scaling region. Nevertheless, it can be seen how the estimator $D_{m}^{U}$ approaches $D$ when the variance among the curves for different $m$ values is the smallest [ $\ln h \approx-0.35$ for Fig. 9(c), $\ln h \approx-0.85$ for Fig. 9(f), and $\ln h \approx-1.2$ for Fig. 9(i)]. $D_{m}^{T}$ displays a similar behavior to $D_{m}^{U}$ even in the presence of noise.

\section{B. Correlation entropy $\left(K_{2}\right)$}

In Appendix $\mathrm{C}$, we propose the following coarse-grained correlation entropy estimator:

$$
K_{m}^{U}(h)=\frac{1}{2 \tau}\left\{-\ln \frac{U_{m+2}^{\beta=m+2}}{U_{m}^{\beta=m}}+\ln \left[\frac{4 \sigma^{2}}{h^{2}+4 \sigma^{2}}\left(\frac{m-\frac{d \ln U_{m}^{\beta=m}(h)}{d \ln h}}{m-D}\right)+\frac{h^{2}}{h^{2}+4 \sigma^{2}}\left(\frac{\frac{d \ln U_{m}^{\beta=m}(h)}{d \ln h}}{m}+1\right)\right]-\ln \left(\frac{D}{m}+1\right)\right\}
$$

As we previously proved, when $\sigma \rightarrow 0$ the quantity $d \ln U_{m}^{\beta=m}(h) / d \ln h \rightarrow D$. Then $K_{m}^{U} \rightarrow \ln (D / m+1)-$ $2 \tau K_{2}$, which is the noiseless correlation entropy functional [Eq. (20)].

In Fig. 10 we present $K_{m}^{U}$ and $K_{m}^{T}$ as functions of $\ln h$ for different $m$ values. These estimators were calculated using time series coming from the Henon map with different noise levels (noiseless, $\sigma=0.05$, and $\sigma=0.2$ ) and a different number of available phase-space vectors $Q=\{500,5000,10000\}$.

In the absence of noise, we can see from Figs. 10(a), 10(d), and $10(\mathrm{~g})$ that $K_{m}^{U}$ oscillates around the reported value of correlation entropy $\left(K_{2}=0.3\right)[11,16]$ regardless of $m$. On the other hand, it can be observed that $K_{m}^{T}$ is unable to converge for the tested $m$ values. It can be seen that the amplitude of the oscillations of $K_{m}^{U}$ is higher for small $h$ values than for larger ones. These oscillations are attenuated as the number of available phase-space vectors is increased.

When the noise level is low $(\sigma=0.05)$, it is shown in the second column of Fig. 10 that $K_{m}^{U}$ still converges to $K_{2}$ for a suitable range of $\ln h$ values. For $Q=500$, this range is $-1.9 \leqslant \ln h \leqslant-1.1$, and for $Q>500$ this range is even larger. For high noise levels $(\sigma=0.2)$ it is observed from
Figs. 10(c), 10(f), and 10(i) that for $K_{m}^{U}$ it is not difficult to find an approximately $h$-independent region. On the contrary, $K_{m}^{T}$ does not converge to $K_{2}$. Moreover, it can be seen that, in contrast to $K_{m}^{T}, K_{m}^{U}$ is not highly dependent on $m$, even in the presence of noise.

\section{Noise level $(\sigma)$}

To propose a coarse-grained estimator of noise level, inspired in [9], we define a noise level functional based on the UCI (see Appendix D):

$$
\begin{aligned}
\Delta_{m}^{U}(h)= & \frac{1}{2}\left[\frac{d \ln U_{m+2}^{\beta=m}}{d \ln h}-\frac{d \ln U_{m}^{\beta=m}}{d \ln h}\right] \\
= & \frac{1}{2}\left[2(m+1) \frac{{ }_{2} F_{1}\left(m+2, \frac{m-D}{2}+1 ; \frac{m+2}{2}+1 ; \frac{-h^{2}}{4 \sigma^{2}}\right)}{{ }_{2} F_{1}\left(m+1, \frac{m-D}{2}+1 ; \frac{m+2}{2}+1 ; \frac{-h^{2}}{4 \sigma^{2}}\right)}\right. \\
& \left.-2 m \frac{{ }_{2} F_{1}\left(m+1, \frac{m-D}{2} ; \frac{m+2}{2} ; \frac{-h^{2}}{4 \sigma^{2}}\right)}{{ }_{2} F_{1}\left(m, \frac{m-D}{2} ; \frac{m+2}{2} ; \frac{-h^{2}}{4 \sigma^{2}}\right)}\right] .
\end{aligned}
$$

It can be shown that $\Delta_{m}^{U}=1$ for $h \rightarrow 0$ and it decreases monotonically to 0 when $h \rightarrow \infty$ (see Appendix D). This 


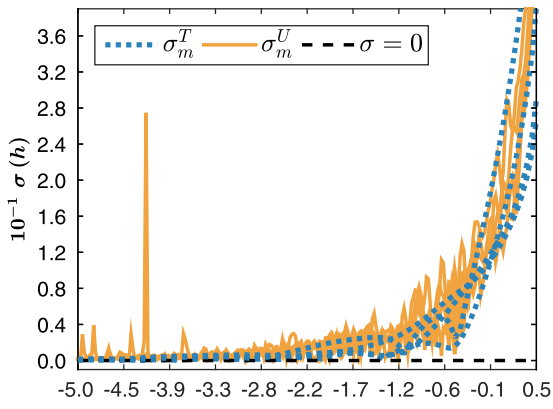

(a)

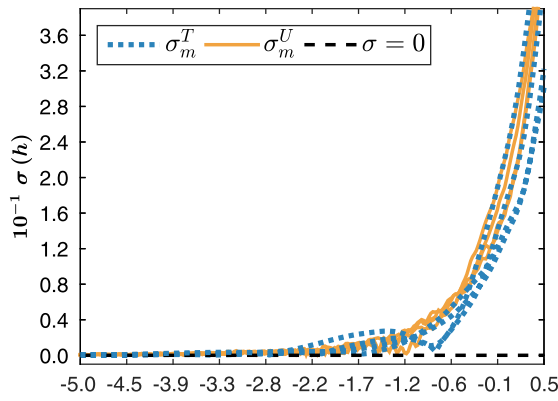

(d)

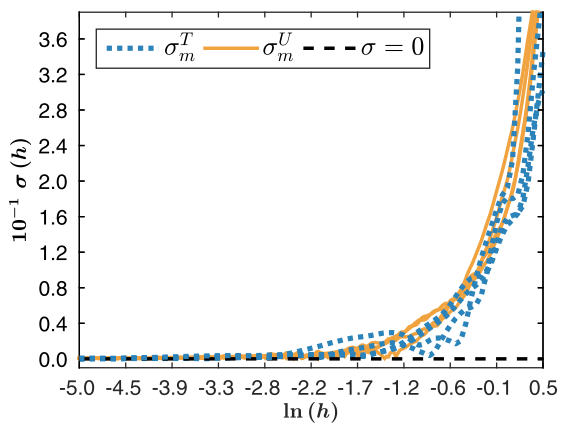

(g)

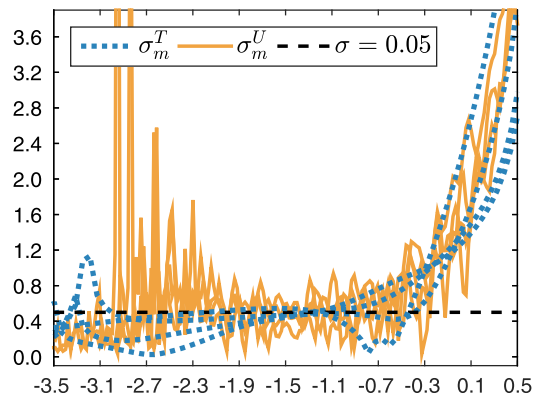

(b)

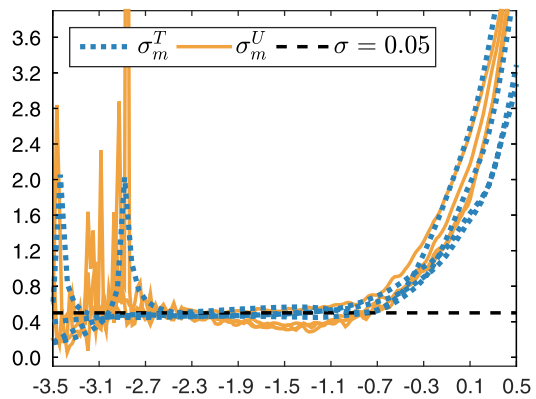

(e)

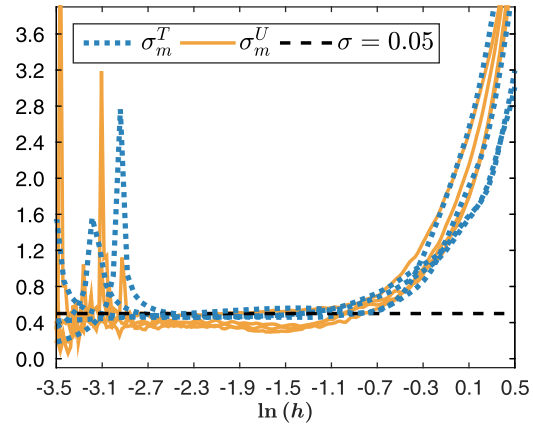

(h)

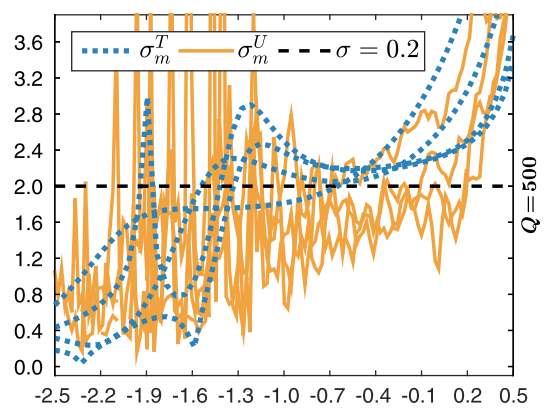

(c)

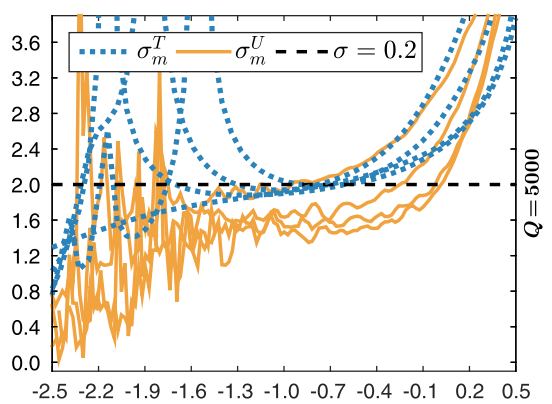

(f)

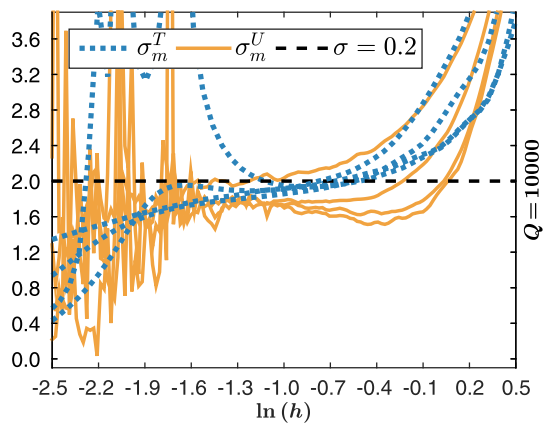

(i)

FIG. 11. Noise level coarse-grained estimators for the Henon map. $\sigma_{m}^{T}$ (dotted blue line) and $\sigma_{m}^{U}$ (solid orange line) for $m=[2,4,6,8]$ and $Q=\{500,5000,10000\}$. Top row: $Q=500$ (a) noiseless, (b) $\sigma=0.05$, and (c) $\sigma=0.2$. Middle row: $Q=5000$ (d) noiseless, (e) $\sigma=0.05$, and (f) $\sigma=0.2$. Bottom row: $Q=10000$ (g) noiseless, (h) $\sigma=0.05$, and (i) $\sigma=0.2$. The true value is shown in each plot (dashed black line).

means that $\Delta_{m}^{U}$ falls off from 1 to 0 on a scale proportional to the noise level $\sigma$. This kind of behavior has been observed in other noise level functionals $[9,13,19,36]$.

For low dimensional systems and large $m$, it can be shown that (see Appendix D)

$$
\Delta_{m}^{U}(z) \approx \frac{1}{1-z},
$$

where $z=-h^{2} / 4 \sigma^{2}$. Finally, the coarse-grained noise level estimator $\sigma_{m}^{U}(h)$ can be calculated as

$$
\sigma_{m}^{U}(h)=\frac{h}{2} \sqrt{\frac{\Delta_{m}^{U}(h)}{1-\Delta_{m}^{U}(h)}} .
$$

Figure 11 shows the noise level estimators $\sigma_{m}^{U}$ and $\sigma_{m}^{T}$ for $m=[2,4,6,8]$. They were obtained using time series from the
Henon map under different noise conditions (noiseless, $\sigma=$ 0.05 , and $\sigma=0.2$ ) and the number of available phase-space vectors $Q=\{500,5000,10000\}$.

In the absence of noise, it is shown in the first column of Fig. 11 that $\sigma_{m}^{U}$ tends to the real noise level value $\sigma=0$ for small $h$ and regardless of the value of $m$ or $Q$. It can be observed how the oscillation of $\sigma_{m}^{U}$ around the real noise level value decreases with increasing $Q$.

For a small noise level $(\sigma=0.05)$, three types of behavior of $\sigma_{m}^{U}$ can be distinguished into three regions of the scale $h$. In the second column of Fig. 11 it can be seen that for small $h$ values, $\sigma_{m}^{U}$ is characterized by a high variance (region I). Next, we can find a range of $h$ at which the variance strongly decreases (region II). Finally, $\sigma_{m}^{U}$ increases rapidly for high values of $h$ (region III). It can be seen that $\sigma_{m}^{U}$ best approaches the real noise level value at region II. Moreover, this behavior is consistent through the different $m$ and $Q$ values. 


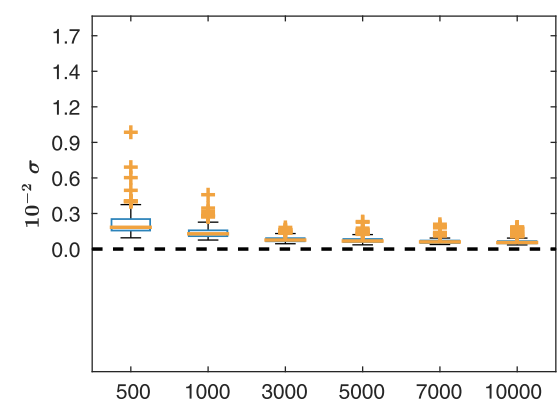

(a)

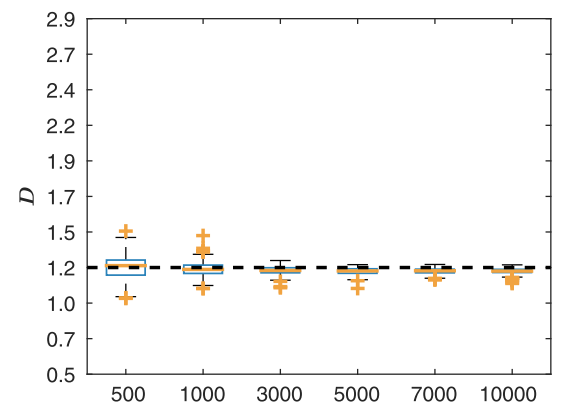

(d)

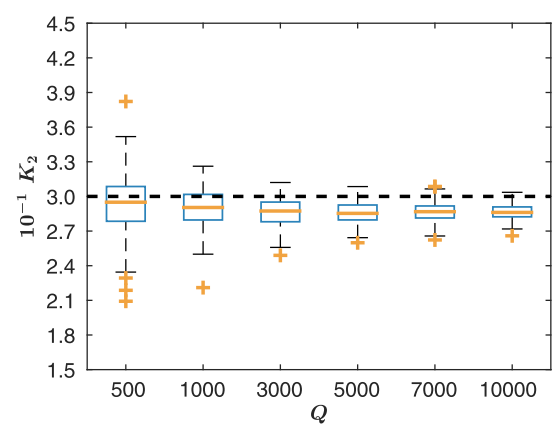

(g)

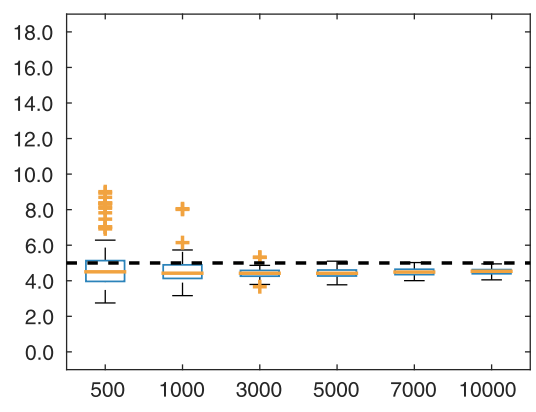

(b)

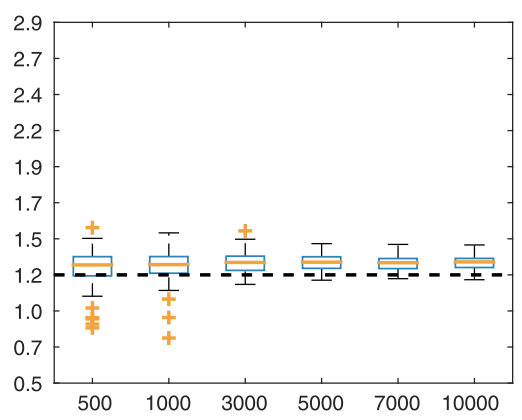

(e)

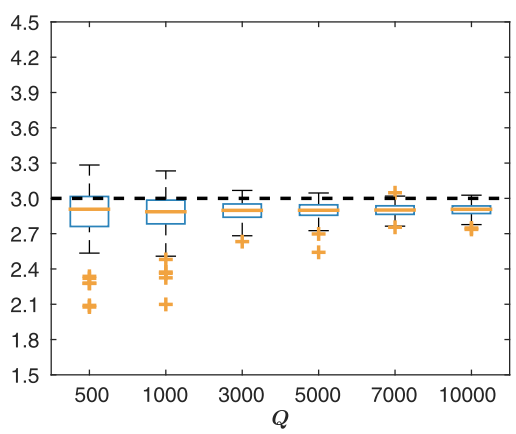

(h)

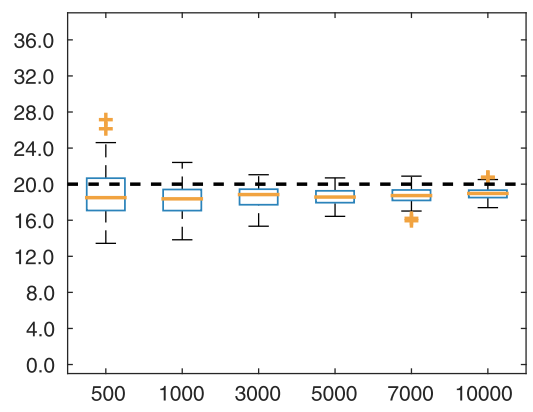

(c)

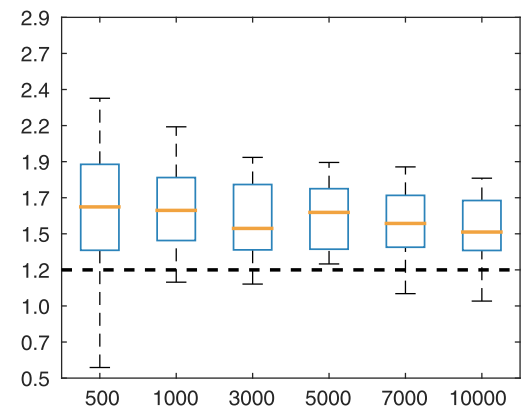

(f)

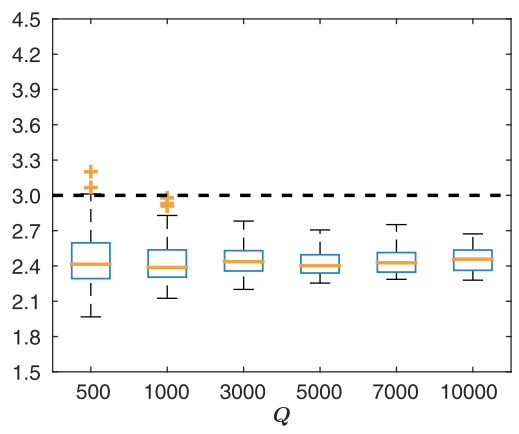

(i)

FIG. 12. Henon map. Box plot of the estimation of $\sigma, D$, and $K_{2}$ for different numbers of available phase-space vectors, $Q=\{500,1000,3000,5000,10000\}$. First row: estimation of $\sigma$. (a) Noiseless, (b) $\sigma=0.05$, and (c) $\sigma=0.2$. Second row: estimation of $D$. (d) Noiseless, (e) $\sigma=0.05$, and (f) $\sigma=0.2$. Third row: estimation of $K_{2}$. (g) Noiseless, (h) $\sigma=0.05$, and (i) $\sigma=0.2$. The reported values are shown by a dashed black line.

For a high noise level $(\sigma=0.2)$, the $h$ dependence of $\sigma_{m}^{U}$ can also be divided into the aforementioned regions [Figs. 11(c), 11(f), and 11(i)]. Moreover, region II still allows us to approximate the real noise level value, although it is shorter than in cases of lower noise. It can be observed that the behavior of $\sigma_{m}^{U}$ is similar to the behavior of $\sigma_{m}^{T}$.

\section{AUTOMATIC INVARIANT ESTIMATION BASED ON THE UCI ${ }^{\beta=m}$}

Based on the observed behavior of the coarse-grained estimators $D_{m}^{U}, K_{m}^{U}$, and $\sigma_{m}^{U}$, we designed a strategy to automatically find the invariants $D, K_{2}$, and $\sigma$. The first step is to estimate the UCIs: $U_{m}^{\beta=m}(h)$ and $U_{m+2}^{\beta=m}(h)$ for different $m \geqslant 2$. Next, we must obtain the coarse-grained estimator $\sigma_{m}^{U}$ [Eq. (25)] using $U_{m}^{\beta=m}(h)$ and $U_{m+2}^{\beta=m}(h)$. To find a range of $h$ from which $\sigma$ can be estimated, we analyze the derivative of $\sigma_{m}^{U}$. We choose the $h$ value at which the absolute value of this derivative is closest to zero as the center of the scale range. Then the noise level is estimated for each $m$ value and $\sigma$ is calculated as the average over these quantities.

Once $\sigma$ is found, we proceed to calculate the correlation dimension $D$. For this goal, we compute the coarse-grained dimension estimator $D_{m}^{U}$ [Eq. (21)] using $\sigma, U_{m}^{\beta=m}(h)$ and $U_{m}^{\beta=m-2}(h)$. To obtain a suitable range of $h$ values, the variance of $D_{m}^{U}$ through $m$ must be computed. This range is centered at the value that minimizes the variance. Then, we calculate the correlation dimension for each $m$ value, and $D$ is estimated as the average over $m$.

Finally, $K_{2}$ can be estimated using $K_{m}^{U}$ [Eq. (22)], calculated with $U_{m}^{\beta=m}(h), U_{m+2}^{\beta=m+2}(h)$, and the previously founded $\sigma$ and 


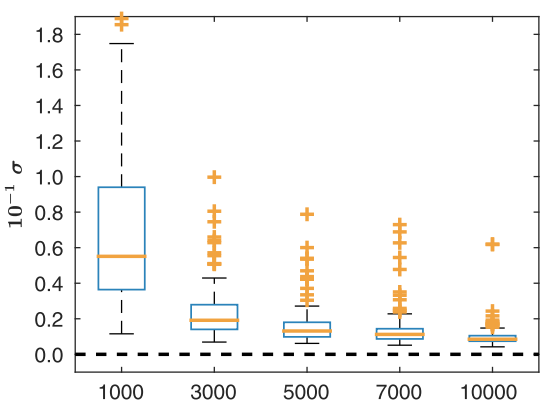

(a)

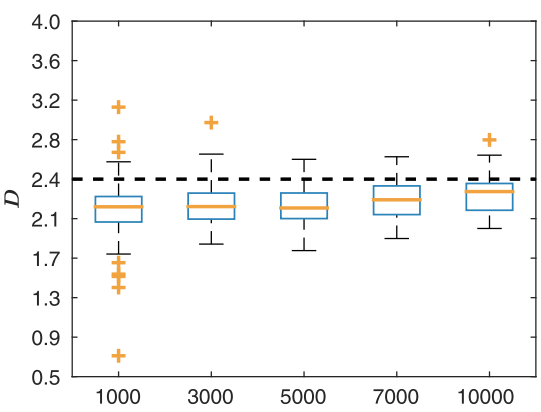

(d)

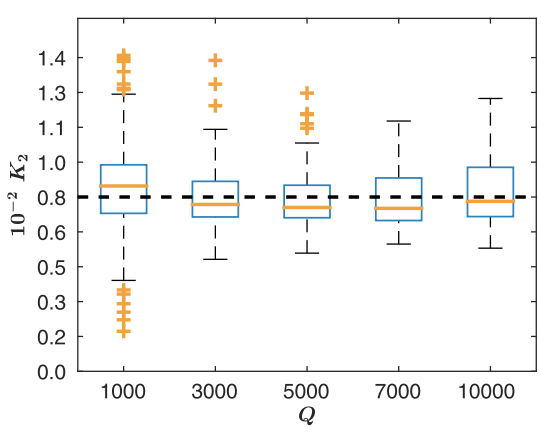

(g)

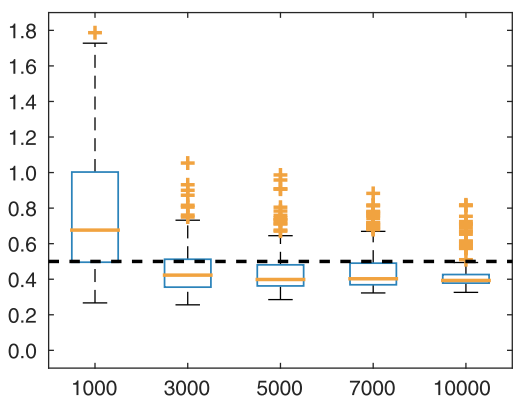

(b)

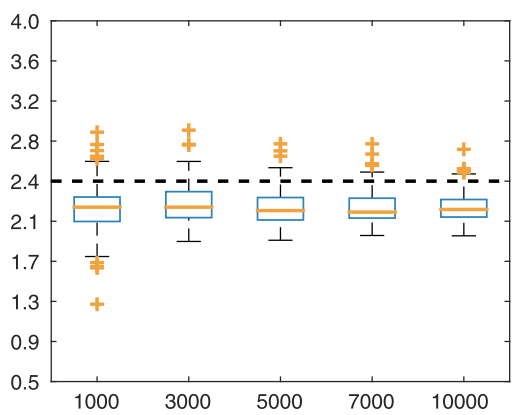

(e)

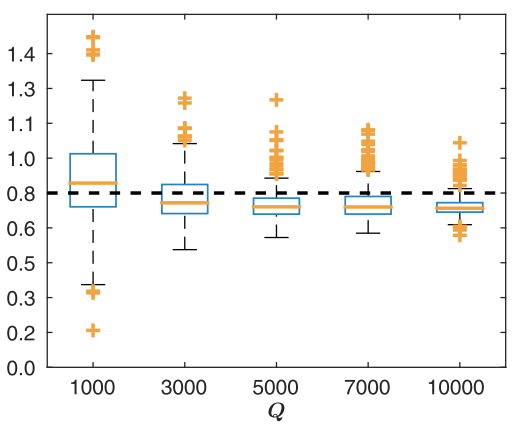

(h)

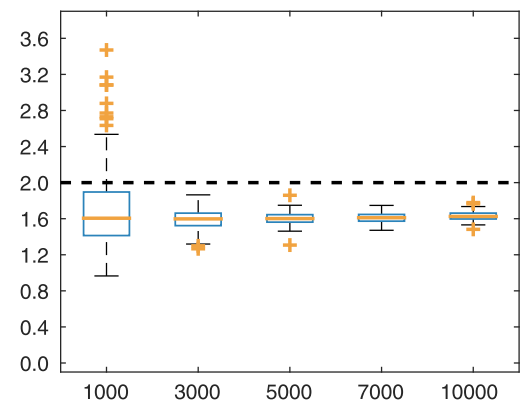

(c)

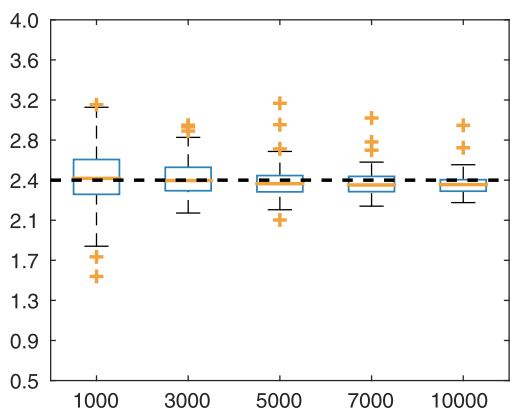

(f)

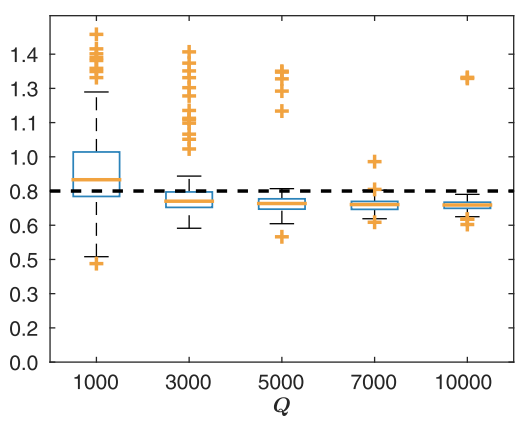

(i)

FIG. 13. Mackey-Glass box plot of the estimation of $\sigma, D$, and $K_{2}$ for different numbers of available phase-space vectors, $Q=\{1000,3000,5000,10000\}$. First row: estimation of $\sigma$. (a) Noiseless, (b) $\sigma=0.05$, and (c) $\sigma=0.2$. Second row: estimation of $D$. (d) Noiseless, (e) $\sigma=0.05$, and (f) $\sigma=0.2$. Third row: estimation of $K_{2}$. (g) Noiseless, (h) $\sigma=0.05$, and (i) $\sigma=0.2$. The reported values are shown by a dashed black line.

$D$. To find a range of $h$ from where $K_{2}$ can be estimated, we analyze the derivative of $K_{m}^{U}$. The $h$ at which the absolute value of this derivative is closest to zero is chosen as the center of the scale range. Finally, the correlation entropy is estimated for each $m$ value, and $K_{2}$ is obtained as the average over $m$. The automatic estimator of invariants is summarized in Algorithm 2.

To evaluate this method, we apply it to 128 Henon map realizations with different noise levels $\sigma=\{0,0.05,0.2\}$. All realizations were normalized (unitary standard deviation), and the UCIs were calculated using $m=\{4,5, \ldots, 8\}$.

In Fig. 12, the box plots of the invariants $D, K_{2}$, and $\sigma$ are shown for different numbers of available phase-space vectors $Q$. The estimation of the noise level $\sigma$ is presented in the first row of Fig. 12. Generally speaking, we can observe that this methodology achieves a reasonable estimation of $\sigma$ even under high noise levels. Nevertheless, $\sigma$ is slightly underestimated for moderate and high noise levels. As is expected, the variance of the estimation decreases as the number of available phasespace vectors is increased.

The estimated correlation dimension $D$ is shown in the second row of Fig. 12. In the absence of noise, Fig. 12(d) shows that the estimations are very close to the reported value $D=1.22$. It can be seen in Fig. 12(e) that for small amounts of noise, $D$ is slightly overestimated. However, for higher noise levels the precision of this estimation decreases [Fig. 12(f)]. As with $\sigma$, the variance of the estimation decreases as the number of available phase-space vectors is increased.

In terms of the correlation entropy $K_{2}$, it can be observed from the third row of Fig. 12 that the methodology proposed 
Algorithm 2 Automatic estimation of invariants using the coarse-grained estimators $\sigma_{m}^{U}(h), D_{m}^{U}(h)$, and $K_{m}^{U}(h)$.

1: Calculate $U_{m}^{\beta=m}(h)$ with Alg. 1 , using noise $\mu_{\omega} \sim \chi_{m}^{2}(h)$.

2: Calculate $U_{m+2}^{\beta=m}(h)$ with Alg. 1, forming phase-space vectors with embedding dimension $(m+2)$ and using noise $\mu_{\omega} \sim \chi_{m}^{2}(h)$. Note that the degrees of freedom of the chi-squared distribution is $m$.

3: Obtain the coarse-grained estimator $\sigma_{m}^{U}(h)$ using Eq. (25). Estimate $\sigma$ within a range of $h$ centered at the $h$ value at which $\left|d \sigma_{m}^{U}(h) / d h\right|$ is closest to zero. Here $|\cdot|$ denotes absolute value.

4: Compute the coarse-grained estimator $D_{m}^{U}(h)$ using Eq. (21) and the value of $\sigma$ found in step 3. Estimate $D$ within a range of $h$ centered at the $h$ value at which the variance of $D_{m}^{U}(h)$ across the different $m$ is minimum.

5: Calculate the coarse-grained estimator $K_{m}^{U}(h)$ using Eq. (22), the value of $\sigma$ found in step 3, and the value of $D$ found in 4 . Estimate $K_{2}$ within a range of $h$ centered at the $h$ value at which $\left|d K_{m}^{U}(h) / d h\right|$ is closest to zero.

herein places the estimation of $K_{2}$ near its reported value $\left(K_{2}=0.3\right)$, at least for small noise levels. Interestingly, Fig. 12(g) shows that, in the absence of noise, the coarsegrained estimator $K_{m}^{U}$ has a median of $K_{2}=0.285$, which is smaller than that reported in the literature.

The good performance in the estimation of $K_{2}$ is kept for small noise levels [Fig. 12(h)]. Nevertheless, for higher $\sigma$ it is difficult to achieve good estimations of $K_{2}$ [Fig. 12(i)], but the estimation is not too far from the reported value.

We also tested our approach using the Mackey-Glass system, whose delay differential equation is

$$
\dot{x}(t)=\frac{a x(t-\lambda)}{1+[x(t-\lambda)]^{10}}-b x(t),
$$

where $a=0.2, b=0.1$, and $\lambda=23$. We calculate the invariants $\sigma, D$, and $K_{2}$ from 128 realizations with different initial conditions and normalized to have unitary standard deviation. The embedding dimension was $m=\{4,6, \ldots, 16\}$ and the embedding lag $\tau$ was set to the one corresponding to the first local minimum of the mutual information function ( $\tau=20$ ) [38]. Moreover, the nearest 15 temporal neighbors of each phase-space vector were discarded [15].

Figure 13 shows the box plots of the estimation of the Mackey-Glass system's invariants for an increasing number of available phase-space vectors, and for noise levels $\sigma=$ $\{0,0.05,0.2\}$. In the first row [Figs. 13(a)-13(c)], it can be observed that for this system it is more difficult to achieve a precise estimation of $\sigma$ than for the Henon map. However, the estimation is acceptable. It can be seen that $\sigma_{m}^{U}$ underestimates $\sigma$ for low and high noise levels [Figs. 13(b) and 13(c)].

The estimation of $D$ is shown in the second row of Fig. 13. For this system, our approach achieves a good estimation of the reported value of its correlation dimension $(D=2.44)$ [39], despite the errors involved in the calculation of $\sigma$. This result is maintained even under high level conditions.

It should be noted that the estimations of the correlation entropy (third row of Fig. 13) are in agreement with the reported value $K_{2}=0.008$ [39], even with inaccuracies in the estimation of $D$ and $\sigma$. Furthermore, this behavior is consistent under noise level $\sigma=0.2$ [Fig. 13(c)].

\section{DISCUSSION}

In this section, we want to recall the most important contributions of this article as well as some technical aspects of the use of the UCI for the estimation of invariants. The idea of the noise-assisted correlation integral, $S_{m}(h)$, occurred to us by observing the similarities between the correlation algorithm (see Fig. 1) proposed by Grassberger et al. and the suprathreshold stochastic resonance model. This idea led us to propose the noise-assisted correlation algorithm (see Fig. 2). One of the main results of this work is Eq. (11). It allows us to define a correlation integral in which the kernel is induced by the noise used in the noise-assisted correlation algorithm.

The NCI has two advantages. The first is the possibility of using different kernel functions besides the Gaussian one. This could help with the analytical modeling of situations that deteriorate due to Gaussianity. The second advantage is related to the calculation of correlation integrals whose kernel functions require numerical integration. If one of these functions can be considered as the complementary cumulative distribution functions of a random variable, then the kernel can be induced by using noise with such complementary cumulative distribution functions, and this could be faster than its numerical evaluation. It is important to notice that the classical correlation integral (by Grassberger and Procaccia) and the GCI (by Diks) can be thought of as particular cases of the NCI. Moreover, it was demonstrated here that the GCI can be achieved by using noise with Rayleigh distribution in the noise-assisted correlation algorithm.

We must recall that the squared Rayleigh distribution is a particular case of the $\chi_{\beta}^{2}$ distribution with degree of freedom $\beta=2$. Based on this idea, we proposed a kernel function using the complementary cumulative distribution functions of the $\chi_{\beta}^{2}$ obtaining the second main result of this article, namely the UCI [Eq. (15)].

We demonstrated that the noise level $\sigma$ as well as the correlation dimension $D$ can be estimated from the UCI. The main advantage over the GCI is that the UCI allows us to introduce information on the embedding dimension $m$ into the kernel function. This action greatly improves the estimation of the correlation entropy $K_{2}$. This estimation, in the absence of noise, is independent of the embedding dimension $m$, in contrast to the estimation-based GCI (see Fig. 7). Additionally, the estimator proposed herein is more robust against noise.

Other important aspect of the UCI is that its equation involves the Gauss hypergeometric function, which in turn includes many other functions as special or limiting cases [40]. Moreover, the Gauss hypergeometric function also has a vast set of identities that allowed us to propose three different coarse-grained estimators: a correlation dimension estimator $D_{m}^{U}$ [Eq. (21)], a correlation entropy estimator $K_{m}^{U}$ [Eq. (22)], and a noise-level estimator $\sigma_{m}^{U}$ [Eq. (25)].

The behavior of the proposed coarse-grained estimator $\left(\sigma_{m}^{U}\right)$ is similar to the one proposed by Nolte et al. $\left(\sigma_{m}^{T}\right)$. Also, the estimators $D_{m}^{U}$ and $D_{m}^{T}$ achieve similar results. On the other 
hand, the estimation of $K_{2}$ is more precise using $K_{m}^{U}$ than $K_{m}^{T}$, as is shown in Fig. 10.

Based on the coarse-grained estimators, we have designed a methodology to automatically calculate the invariants of a system. We have found that the reliability of the estimation of $D$ and $K_{2}$ depends on the accuracy of the estimation of $\sigma$. The results of this methodology can be seen in Figs. 12 and 13.

The use of a wavelet transform approach to approximate the derivative [37] allows us to achieve better estimations of the invariants. This is because it implements a low-pass filter reducing the high-frequency oscillations that are naturally present in the coarse-grained estimators derived from the UCI.

Another way to mitigate the high-frequency oscillations of the coarse-grained estimators $D_{m}^{U}, K_{m}^{U}$, and $\sigma_{m}^{U}$ is to increase the number of available phase-space vectors, which can be done in two ways. The first is to increase the time-series length, which is not always possible. The second option is to calculate all the distances or squared distances between phase-space vectors and make copies of them. As a result, the number of comparators in the noise-assisted algorithm would be increased. Then, different realizations of noise must be added to each copy, followed by the thresholding procedure. Finally, the noise-assisted correlation sum, $\widehat{S}(h)$, must be computed as the average of all comparator outputs. In other words, the idea is to replicate several times the diagram presented in Fig. 2. The smoothness of the estimation and the computational effort will be increased with the number of copies.

\section{CONCLUSIONS}

In this study, we have presented the foundations of the noise-assisted correlation integral as well as the algorithm to calculate it. The $U$-correlation integral was derived as a special case of the noise-assisted correlation integral. The UCI has the particularity that its kernel function incorporates information of the embedding dimension. From this correlation integral, we have deduced coarse-grained estimators for $D, K_{2}$, and $\sigma$. We have studied the behavior of these estimators under different noise conditions and data lengths. From these results, we can conclude that the incorporation of information about the embedding dimension is useful for the estimation of $K_{2}$. Based on the coarse-grained functions proposed herein, we designed a methodology to automatically calculate the system's invariants. This approach has yielded reliable estimations of $D, K_{2}$, and $\sigma$ even under high noise levels.

The NCI can lead to the exploration of new kernel functions. This will allow us to derive new correlation integrals that could improve the estimation of system invariants, increasing our capacity to analyze and understand different natural phenomena.

\section{ACKNOWLEDGMENTS}

This work was supported by the National Scientific and Technical Research Council (CONICET) of Argentina, the National University of Entre Ríos (UNER), CITER, and Grants PID-6136 (UNER), PICT-2012-2954 (ANPCyTUNER), and PIO-14620140100014CO (CITER-CONICET).

\section{APPENDIX A: LIMIT BEHAVIOR OF $d \ln U_{m}^{\beta=m}(h) / d \ln h$}

To analyze the limit behavior of the function $d \ln U_{m}^{\beta=m}(h) / d \ln h$, we must define

$$
\begin{aligned}
& a=\frac{\beta+m}{2}, \quad b=\frac{m-D}{2}, \quad c=\frac{m+2}{2}, \\
& z=-\frac{h^{2}}{4 \sigma^{2}}, \quad \frac{d z}{d h}=-\frac{h}{2 \sigma^{2}},
\end{aligned}
$$

and

$$
P=(-1)^{m / 2} \frac{\widehat{\phi}}{2}(2 \sigma)^{D} \frac{\Gamma(D / 2)}{\Gamma(\beta / 2)} \frac{\Gamma((m+\beta) / 2)}{\Gamma(m / 2+1)} e^{-m \tau K_{2}} .
$$

Equation (15) can then be rewritten as

$$
U_{m}^{\beta}(z)=P z^{m / 2}{ }_{2} F_{1}(a, b ; c ; z) .
$$

We can calculate the derivative of $U_{m}^{\beta}(z)$ with respect to $h$ as

$$
\frac{d}{d h} U_{m}^{\beta}(z)=\frac{d}{d z}\left[P z^{m / 2}{ }_{2} F_{1}(a, b ; c ; z)\right] \frac{d z}{d h} .
$$

Using Eq. 15.2.1 from Ref. [41],

$$
\begin{aligned}
\frac{d}{d h} U_{m}^{\beta}(z)= & \frac{-P h}{2 \sigma^{2}} z^{m / 2}\left[\frac{m}{2 z}{ }_{2} F_{1}(a, b ; c ; z)\right. \\
& \left.+\frac{a b}{c}{ }_{2} F_{1}(a+1, b+1 ; c+1 ; z)\right] .
\end{aligned}
$$

The logarithmic derivative of $U_{m}^{\beta}(z)$ is obtained as

$$
\begin{aligned}
\frac{d \ln U_{m}^{\beta}(z)}{d \ln h}= & \frac{h}{U_{m}^{\beta}(z)} \frac{d}{d h} U_{m}^{\beta}(z) \\
= & \frac{2 P z^{m / 2+1}}{P z^{m / 2}{ }_{2} F_{1}(a, b ; c ; z)}\left[\frac{m}{2 z}{ }_{2} F_{1}(a, b ; c ; z)\right. \\
& \left.+\frac{a b}{c}{ }_{2} F_{1}(a+1, b+1 ; c+1 ; z)\right] \\
= & m+\frac{2 a b z}{c} \frac{{ }_{2} F_{1}(a+1, b+1 ; c+1 ; z)}{{ }_{2} F_{1}(a, b ; c ; z)} .
\end{aligned}
$$

Other expressions for the logarithmic derivative of $U_{m}^{\beta}(z)$ can be found using the identities of the Gauss hypergeometric function. For example, from Eq. (A2) and using $(z b / c){ }_{2} F_{1}(a+1, b+1 ; c+1 ; z)={ }_{2} F_{1}(a+1, b ; c ; z)-$ ${ }_{2} F_{1}(a, b ; c ; z)$, it can be shown that

$$
\frac{d \ln U_{m}^{\beta}(z)}{d \ln h}=2 a \frac{{ }_{2} F_{1}(a+1, b ; c ; z)}{{ }_{2} F_{1}(a, b ; c ; z)}-\beta .
$$

For very noisy time series $(\sigma \gg h), z \rightarrow 0$. Taking into account that ${ }_{2} F_{1}(a, b ; c ; 0)=1$, it can be proven from Eq. (A3) that

$$
\lim _{z \rightarrow 0} \frac{d \ln U_{m}^{\beta}(z)}{d \ln h}=m
$$


When the time series is noise-free or has a relatively small amount of noise $(\sigma \ll h)$, the value of $z \rightarrow-\infty$. By making use of Eq. 15.3.7 from Ref. [41], and the fact that $1 / z \rightarrow 0$, we can obtain

$$
\begin{aligned}
{ }_{2} F_{1}(a, b ; c ; z) & =\frac{\Gamma(c) \Gamma(b-a)}{\Gamma(b) \Gamma(c-a)}(-z)^{-a}{ }_{2} F_{1}(a, 1-c+a ; 1-b+a ; 1 / z)+\frac{\Gamma(c) \Gamma(a-b)}{\Gamma(a) \Gamma(c-b)}(-z)^{-b}{ }_{2} F_{1}(b, 1-c+b ; 1-a+b ; 1 / z) \\
& =\frac{\Gamma(c) \Gamma(b-a)}{\Gamma(b) \Gamma(c-a)}(-z)^{-a}+\frac{\Gamma(c) \Gamma(a-b)}{\Gamma(a) \Gamma(c-b)}(-z)^{-b} .
\end{aligned}
$$

We can then rewrite the limit when $z \rightarrow-\infty$ of Eq. (A2) as

$$
\lim _{z \rightarrow-\infty} \frac{d \ln U_{m}^{\beta}(z)}{d \ln h}=m-\frac{2 a b}{c} \frac{\frac{\Gamma(c+1) \Gamma(b-a)}{\Gamma(b+1) \Gamma(c-a)}(-z)^{b-a}+\frac{\Gamma(c+1) \Gamma(a-b)}{\Gamma(a+1) \Gamma(c-b)}}{\frac{\Gamma(c) \Gamma(b-a)}{\Gamma(b) \Gamma(c-a)}(-z)^{b-a}+\frac{\Gamma(c) \Gamma(a-b)}{\Gamma(a) \Gamma(c-b)}} .
$$

Finally, taking into account that $a>b$, we find that

$$
\lim _{z \rightarrow-\infty} \frac{d \ln U_{m}^{\beta}(z)}{d \ln h}=m-\frac{2 a b}{c} \frac{\frac{\Gamma(c+1) \Gamma(a-b)}{\Gamma(a+1) \Gamma(c-b)}}{\frac{\Gamma(c) \Gamma(a-b)}{\Gamma(a) \Gamma(c+b)}}=m-2(a b / c)(c / a)=D .
$$

\section{APPENDIX B: DEDUCTION OF $D_{m}^{U}(h)$}

Using Eq. 9.137-9 from Ref. [42], we obtain

$$
\frac{a b z}{c} \frac{{ }_{2} F_{1}(a+1, b+1 ; c+1 ; z)}{{ }_{2} F_{1}(a, b ; c ; z)}=\frac{c-a}{1-z} \frac{{ }_{2} F_{1}(a-1, b ; c ; z)}{{ }_{2} F_{1}(a, b ; c ; z)}-\frac{c-a-b z}{1-z},
$$

and using Eq. (A2), we can show that

$$
\frac{d U_{m}^{\beta}(z)}{d \ln h}=m+\frac{2(c-a)}{1-z} \frac{{ }_{2} F_{1}(a-1, b ; c ; z)}{{ }_{2} F_{1}(a, b ; c ; z)}-\frac{2(c-a-b z)}{1-z} .
$$

We need to find an estimator for the quotient ${ }_{2} F_{1}(a-1, b ; c ; z) /{ }_{2} F_{1}(a, b ; c ; z)$. Using Eqs. (15) and (A1), we define the estimator as

$$
\frac{U_{m}^{\beta-2}(z)}{U_{m}^{\beta}(z)}=\frac{\beta-2}{2(a-1)} \frac{{ }_{2} F_{1}(a-1, b ; c ; z)}{{ }_{2} F_{1}(a, b ; c ; z)} .
$$

Using Eq. (B2) in Eq. (B1), we get

$$
\frac{d \ln U_{m}^{\beta}(z)}{d \ln h}=m+\frac{4(c-a)(a-1)}{(1-z)(\beta-2)} \frac{U_{m}^{\beta-2}}{U_{m}^{\beta}}-\frac{2(c-a-b z)}{1-z} .
$$

Clearing for $b$,

$$
2 b=\frac{1-z}{z}\left[\frac{d \ln U_{m}^{\beta}(z)}{d \ln h}-m\right]-\frac{4(a-1)(c-a)}{z(\beta-2)} \frac{U_{m}^{\beta-2}(z)}{U_{m}^{\beta}(z)}+\frac{2(c-a)}{z} .
$$

Substituting the values of $a, b, c$, and $z$ [Eq. (A1)], setting $\beta=m$, and clearing for $D$, we can define our coarse-grained estimator $D_{m}^{u}(h)$ :

$$
D_{m}^{u}(h)=\left(\frac{4 \sigma^{2}+h^{2}}{h^{2}}\right) \frac{d \ln U_{m}^{\beta=m}(h)}{d \ln h}+\frac{4 \sigma^{2}}{h^{2}}(2 m-2)\left[\frac{U_{m}^{\beta=m-2}(h)}{U_{m}^{\beta=m}(h)}-1\right] .
$$

It is necessary to clarify that the estimator $U_{m}^{\beta=m-2}(h)$ should be understood as the UCI calculated with phase-space vectors of dimension $m$ and noise dimension $m-2$.

\section{APPENDIX C: DEDUCTION OF $K_{m}^{U}(h)$}

The deduction of a coarse-grained estimator for the correlation entropy $K_{2}$ under the UCI involves the use of the estimators $U_{m}^{\beta}(h)$ and $U_{m+2}^{\beta+2}(h)$. First we need to define

$$
M=\frac{\widehat{\phi}}{2}(2 \sigma)^{D} \Gamma(D / 2) .
$$


Using Eqs. (A1) and (C1), we can rewrite Eq. (15) as

$$
U_{m}^{\beta}(z)=M \frac{\Gamma(a) e^{-m \tau K_{2}}(-z)^{m / 2}}{\Gamma(\beta / 2) \Gamma(c)}{ }_{2} F_{1}(a, b ; c ; z),
$$

and we write the quotient $U_{m+2}^{\beta+2}(z) / U_{m}^{\beta}(z)$ as

$$
\begin{aligned}
\frac{U_{m+2}^{\beta+2}(z)}{U_{m}^{\beta}(z)} & =\left[\frac{\Gamma(a+2) \Gamma\left(\frac{\beta}{2}\right) \Gamma(c)}{\Gamma(a) \Gamma\left(\frac{\beta+2}{2}\right) \Gamma(c+1)}\right]\left[\frac{(-z){ }_{2} F_{1}(a+2, b+1 ; c+1 ; z)}{{ }_{2} F_{1}(a, b ; c ; z)} e^{-2 \tau K_{2}}\right] \\
& =\frac{2 a(a+1)}{\beta} \frac{(-z){ }_{2} F_{1}(a+2, b+1 ; c+1 ; z)}{c_{2} F_{1}(a, b ; c ; z)} e^{-2 \tau K_{2}} .
\end{aligned}
$$

Setting $w=a+1$, the last equation can be expressed as

$$
\frac{U_{m+2}^{\beta+2}(z)}{U_{m}^{\beta}(z)}=-\frac{2(w-1)}{\beta} \frac{w z_{2} F_{1}(w+1, b+1 ; c+1 ; z)}{c_{2} F_{1}(w-1, b ; c ; z)} e^{-2 \tau K_{2}} .
$$

Using Eq. 9.137-9 from Ref. [42], we obtain

$$
\frac{b w(z-1) z_{2} F_{1}(w+1, b+1 ; c+1 ; z)}{c_{2} F_{1}(w-1, b ; c ; z)}=(c-w)-(c-w-b z) \frac{{ }_{2} F_{1}(w, b ; c ; z)}{{ }_{2} F_{1}(w-1, b ; c ; z)} .
$$

Using Eq. (C5) in Eq. (C4), we can show that

$$
\frac{U_{m+2}^{\beta+2}(z)}{U_{m}^{\beta}(z)}=\frac{-2(w-1)}{\beta b(1-z)}\left[c-w-(c-w-b z) \frac{{ }_{2} F_{1}(w, b ; c ; z)}{{ }_{2} F_{1}(w-1, b ; c ; z)}\right] e^{-2 \tau K_{2}} .
$$

Replacing $a=w-1$ and knowing that $c-a-1=-\beta / 2$ [Eq. (A1)], we can then write

$$
\frac{U_{m+2}^{\beta+2}(z)}{U_{m}^{\beta}(z)}=\frac{-2 a}{\beta b(1-z)}\left[\left(\frac{\beta}{2}+b z\right) \frac{{ }_{2} F_{1}(a+1, b ; c ; z)}{{ }_{2} F_{1}(a, b ; c ; z)}-\frac{\beta}{2}\right] e^{-2 \tau K_{2}} .
$$

Using Eq. (A3) in Eq. (C7), we prove that

$$
\begin{aligned}
\frac{U_{m+2}^{\beta+2}(z)}{U_{m}^{\beta}(z)} & =\frac{e^{-2 \tau K_{2}}}{\beta b(1-z)}\left\{\frac{2 a \beta}{2}-\left(\frac{\beta}{2}+b z\right)\left[\frac{d \ln U_{m}^{\beta}(z)}{d \ln h}+\beta\right]\right\} \\
& =\frac{e^{-2 \tau K_{2}}}{(1-z)}\left\{\frac{1}{2 b}\left[2 a-\frac{d \ln U_{m}^{\beta}(z)}{d \ln h}-\beta\right]-\frac{z}{\beta}\left[\frac{d \ln U_{m}^{\beta}(z)}{d \ln h}+\beta\right]\right\} .
\end{aligned}
$$

Restoring the values of $a, b$, and $z$, we have

$$
\frac{U_{m+2}^{\beta+2}(h)}{U_{m}^{\beta}(h)}=e^{-2 \tau K_{2}}\left[\frac{4 \sigma^{2}}{h^{2}+4 \sigma^{2}}\left(\frac{m-\frac{d \ln U_{m}^{\beta}(h)}{d \ln h}}{m-D}\right)+\frac{h^{2}}{h^{2}+4 \sigma^{2}}\left(\frac{\frac{d \ln U_{m}^{\beta}(h)}{d \ln h}}{\beta}+1\right)\right] .
$$

Setting $\beta=m$, taking the logarithm on both sides, and allowing for $2 \tau K_{2}$, we can write

$$
2 \tau K_{2}=-\ln \frac{U_{m+2}^{\beta=m+2}(h)}{U_{m}^{\beta=m}(h)}+\ln \left[\frac{4 \sigma^{2}}{h^{2}+4 \sigma^{2}}\left(\frac{m-\frac{d \ln U_{m}^{\beta=m}(h)}{d \ln h}}{m-D}\right)+\frac{h^{2}}{h^{2}+4 \sigma^{2}}\left(\frac{\frac{d \ln U_{m}^{\beta=m}(h)}{d \ln h}}{m}+1\right)\right] .
$$

Subtracting $\ln (D / m+1)$ from both sides of the preceding equation gives

$2 \tau K_{2}-\ln \left(\frac{D}{m}+1\right)=-\ln \frac{U_{m+2}^{\beta=m+2}(h)}{U_{m}^{\beta=m}(h)}+\ln \left[\frac{4 \sigma^{2}}{h^{2}+4 \sigma^{2}}\left(\frac{m-\frac{d \ln U_{m}^{\beta=m}(h)}{d \ln h}}{m-D}\right)+\frac{h^{2}}{h^{2}+4 \sigma^{2}}\left(\frac{\frac{d \ln U_{m}^{\beta=m}(h)}{d \ln h}}{m}+1\right)\right]-\ln \left(\frac{D}{m}+1\right)$.

Finally, from Eq. (C8) we propose a correlation entropy coarse-grained estimator $K_{m}^{U}$ as

$$
K_{m}^{U}(h)=\frac{1}{2 \tau}\left\{-\ln \frac{U_{m+2}^{\beta=m+2}(h)}{U_{m}^{\beta=m}(h)}+\ln \left[\frac{4 \sigma^{2}}{h^{2}+4 \sigma^{2}}\left(\frac{m-\frac{d \ln U_{m}^{\beta=m}(h)}{d \ln h}}{m-D}\right)+\frac{h^{2}}{h^{2}+4 \sigma^{2}}\left(\frac{\frac{d \ln U_{m}^{\beta=m}(h)}{d \ln h}}{m}+1\right)\right]-\ln \left(\frac{D}{m}+1\right)\right\} .
$$




\section{APPENDIX D: DEDUCTION OF $\sigma_{m}^{U}(h)$}

Based on [9], we propose the functional

$$
\Delta_{n, m}^{U}(h)=\frac{1}{n-m}\left[\frac{d \ln U_{n}^{\beta=n-2}(h)}{d \ln h}-\frac{d \ln U_{m}^{\beta=m}(h)}{d \ln h}\right] .
$$

Setting $n=m+2$ and using Eq. (A3), we can write Eq. (D1) as

$$
\Delta_{m}^{U}(z)=\frac{1}{2}\left\{2(m+1) \frac{{ }_{2} F_{1}(m+2, \bar{b} ; \bar{c} ; z)}{{ }_{2} F_{1}(m+1, \bar{b} ; \bar{c} ; z)}-2 m \frac{{ }_{2} F_{1}(m+1, b ; c ; z)}{{ }_{2} F_{1}(m, b ; c ; z)}\right\},
$$

where $\bar{b}=(m+2-D) / 2, \bar{c}=(m+4) / 2, b=(m-D) / 2, c=(m+2) / 2$, and $z=-h^{2} / 4 \sigma^{2}$.

When $h \rightarrow 0, z \rightarrow 0$ and

$$
\lim _{z \rightarrow 0} \Delta_{m}^{U}(z)=\frac{1}{2}[2(m+1)-2 m]=1 .
$$

On the other hand, when $z \rightarrow-\infty \quad(h \rightarrow \infty)$ it can be proven using Eq. 15.3.7 from Ref. [41] that ${ }_{2} F_{1}(a+1, b ; c ; z) /{ }_{2} F_{1}(a, b ; c ; z)=(a-b) / a$. Then

$$
\lim _{z \rightarrow 0} \Delta_{m}^{U}(z)=\frac{1}{2}\left\{2\left[m+1-\frac{m+2-D}{2}\right]-2\left[m-\frac{m-D}{2}\right]\right\}=0 .
$$

For low dimensional systems and large $m$ values, $\bar{b} \approx \bar{c}$ and $b \approx c$. Then taking into account that ${ }_{2} F_{1}(a, b ; b ; z)=(1-z)^{-a}$, Eq. (D2) can be reduced to

$$
\Delta_{m}^{U}(z) \approx \frac{1}{2}\left[\frac{2(m+1)}{1-z}-\frac{2 m}{1-z}\right] \approx \frac{1}{1-z} .
$$

It can be assessed that the limits in which $z \rightarrow 0$ and $z \rightarrow-\infty$ in Eq. (D4) are 1 and 0 , respectively.

[1] S. H. Strogatz, Nonlinear Dynamics and Chaos: With Applications to Physics, Biology, Chemistry, and Engineering (Westview, Cambridge, MA, 2014).

[2] M. Faggini, Chaotic time series analysis in economics: Balance and perspectives, Chaos 24, 042101 (2014).

[3] P. Grassberger and I. Procaccia, Characterization of Strange Attractors, Phys. Rev. Lett. 50, 346 (1983).

[4] P. Grassberger and I. Procaccia, Estimation of the Kolmogorov entropy from a chaotic signal, Phys. Rev. A 28, 2591 (1983).

[5] D. Yang, P. Yang, and C. Zhang, Chaotic characteristic analysis of strong earthquake ground motions, Int. J. Bifurcation Chaos 22, 1250045 (2012).

[6] P. Zou, Q.-X. Li, and N. Wu, Nonlinear analysis of the long-term behavior of solar filaments, Mon. Not. R. Astron. Soc. 437, 38 (2014).

[7] B. Hosseinifard, M. H. Moradi, and R. Rostami, Classifying depression patients and normal subjects using machine learning techniques and nonlinear features from EEG signal, Comput. Methods Programs Biomed. 109, 339 (2013).

[8] P. Grassberger and I. Procaccia, Measuring the strangeness of strange attractors, Physica D 9, 189 (1983).

[9] T. Schreiber, Determination of the noise level of chaotic time series, Phys. Rev. E 48, R13 (1993).

[10] T. Schreiber, Influence of Gaussian noise on the correlation exponent, Phys. Rev. E 56, 274 (1997).

[11] C. Diks, Estimating invariants of noisy attractors, Phys. Rev. E 53, R4263 (1996).

[12] M. Small, Applied Nonlinear Time Series Analysis: Applications in Physics, Physiology and Finance (World Scientific, Singapore, 2005).
[13] C. Diks, Nonlinear Time Series Analysis: Methods and Applications, Nonlinear Time Series and Chaos (World Scientific, Singapore, 1999).

[14] T. Schreiber and H. Kantz, Observing and predicting chaotic signals: Is $2 \%$ noise too much? in Predictability of Complex Dynamical Systems, edited by Y. A. Kravtsov and J. B. Kadtke (Springer, Berlin, 1996), pp. 43-65.

[15] H. Kantz and T. Schreiber, Nonlinear Time Series Analysis (Cambridge University Press, Cambridge, 2004).

[16] D. Yu, M. Small, R. G. Harrison, and C. Diks, Efficient implementation of the Gaussian kernel algorithm in estimating invariants and noise level from noisy time series data, Phys. Rev. E 61, 3750 (2000).

[17] M. Frank, H.-R. Blank, J. Heindl, M. Kaltenhäuser, H. Köchner, W. Kreische, N. Müller, S. Poscher, R. Sporer, and T. Wagner, Improvement of K2-entropy calculations by means of dimension scaled distances, Physica D 65, 359 (1993).

[18] J.-M. Ghez, E. Orlandini, M.-C. Tesi, and S. Vaienti, Dynamical integral transform on fractal sets and the computation of entropy, Physica D 63, 282 (1993).

[19] G. Nolte, A. Ziehe, and K.-R. Müller, Noise robust estimates of correlation dimension and $K_{2}$ entropy, Phys. Rev. E 64, 016112 (2001).

[20] D. Kugiumtzis, Correction of the correlation dimension for noisy time series, Int. J. Bifurcation Chaos 07, 1283 (1997).

[21] J. C. Schouten, F. Takens, and C. M. van den Bleek, Estimation of the dimension of a noisy attractor, Phys. Rev. E 50, 1851 (1994).

[22] A. W. Jayawardena, P. Xu, and W. K. Li, A method of estimating the noise level in a chaotic time series, Chaos 18, 023115 (2008). 
[23] A. W. Jayawardena, P. Xu, and W. K. Li, Modified correlation entropy estimation for a noisy chaotic time series, Chaos (Woodbury, NY) 20, 023104 (2010).

[24] W. R. Bennett, Spectra of quantized signals, Bell Syst. Technol. J. 27, 446 (1948).

[25] L. Gammaitoni, Stochastic resonance and the dithering effect in threshold physical systems, Phys. Rev. E 52, 4691 (1995).

[26] L. Gammaitoni, P. Hänggi, P. Jung, and F. Marchesoni, Stochastic resonance, Rev. Mod. Phys. 70, 223 (1998).

[27] M. D. McDonnell and D. Abbott, What is stochastic resonance? Definitions, misconceptions, debates, and its relevance to biology, PLoS Comput. Biol. 5, e1000348 (2009).

[28] D. Rousseau and F. Chapeau-Blondeau, Suprathreshold stochastic resonance and signal-to-noise ratio improvement in arrays of comparators, Phys. Lett. A 321, 280 (2004).

[29] F. Chapeau-Blondeau, S. Blanchard, and D. Rousseau, Fisher information and noise-aided power estimation from one-bit quantizers, Digital Sign. Proc. 18, 434 (2008).

[30] F. Chapeau-Blondeau, Stochastic resonance and optimal detection of pulse trains by threshold devices, Digital Sign. Proc. 9, 162 (1999).

[31] D. Rousseau and F. Chapeau-Blondeau, Constructive role of noise in signal detection from parallel arrays of quantizers, Sign. Proc. 85, 571 (2005).

[32] D. Rousseau and F. Chapeau-Blondeau, Proceedings International Conference on Complex Systems, Intelligence and Modern Technology Applications, Cherbourg, France (SEE, Paris, 2004), pp. 192-197.
[33] D. Rousseau and F. Chapeau-Blondeau, Noise-improved Bayesian estimation with arrays of one-bit quantizers, IEEE Trans. Instr. Meas. 56, 2658 (2007).

[34] H. Oltmans and P. J. T. Verheijen, Influence of noise on power-law scaling functions and an algorithm for dimension estimations, Phys. Rev. E 56, 1160 (1997).

[35] R. L. Smith, Estimating dimension in noisy chaotic time series, J. R. Stat. Soc.: Ser. B (Methodol.) 54, 329 (1992).

[36] G. Çoban, A. H. Büyüklü, and A. Das, A linearization based non-iterative approach to measure the Gaussian noise level for chaotic time series, Chaos, Solitons Fractals 45, 266 (2012).

[37] J. W. Luo, J. Bai, and J. H. Shao, Application of the wavelet transforms on axial strain calculation in ultrasound elastography, Progr. Nat. Sci. 16, 942 (2006).

[38] F. Kaffashi, R. Foglyano, C. G. Wilson, and K. A. Loparo, The effect of time delay on approximate and sample entropy calculations, Physica D 237, 3069 (2008).

[39] P. Grassberger and I. Procaccia, Dimensions and entropies of strange attractors from a fluctuating dynamics approach, Physica D 13, 34 (1984).

[40] A. Plastino and M. C. Rocca, Hypergeometric foundations of Fokker-Planck-like equations, Phys. Lett. A 380, 1900 (2016).

[41] M. Abramowitz and I. A. Stegun, Handbook of Mathematical Functions: With Formulas, Graphs, and Mathematical Tables, Applied Mathematics Series (Martino Publishing, Mansfield Center, CT, 2014).

[42] I. S. Gradshteyn and I. M. Ryzhik, Table of Integrals, Series, and Products, 5th ed. (Academic Press, San Diego, CA, 1994). 